\title{
On the Diffraction and Reflection of Waves and Pulses by Wedges and Corners
}

\author{
F. Oberhettinger ${ }^{1}$
}

\begin{abstract}
Various problems arising in the theory of the excitation of a perfectly reflecting wedge or corner by a plane, cylindrical, or spherical wave, are dealt with. The incident wave is represented by a line source (acoustic or electromagnetic) parallel to the edge. The spherical wave is emitted by an acoustic point source or by a Hertz dipole with its axis parallel to the edge. The case of an incident plane wave field is obtained as the limiting case (for large distances of the source from the edge) of the cylindrical or spherical wave excitation.
\end{abstract}

\section{Introduction}

Sommerfeld's classical problem of the excitation of a perfectly reflecting half plane by a plane (acoustic or electromagnetic) wave field is based upon the theory of multivalued solutions of the wave equation [34]. ${ }^{2}$ Subsequently this method was extended to the problem of the diffraction of cylindrical or spherical waves by a half plane [3] or by a wedge [44] (in the latter case also for a plane incident wave). Here the incident cylindrical wave is emitted by a line source parallel to the edge (of the half plane or wedge) and the spherical wave is emitted by a point source (in the acoustic case) or by a Hertz dipole (in the electromagnetic case) parallel to the edge. The solutions for the half plane problem are given in the form of integral expressions involving a real variable, while the corresponding wedge problem solutions are given as contour integrals.

The problems described above have been treated ever since with the aid of various techniques. The wedge problem as treated by MacDonald [22] requires the conventional methods of nineteenth century mathematical physics only. Here the solution is given by an infinite series and this is transformed into Wiegrefe's complex integral. For the special case of a half plane (angle of the wedge equal to $2 \pi$ ), reductions to integrals involving a real variable are given. Another approach is a generalization of the method of the images [1, 16, 43].

At first the solution for a wedge of an angle $\pi / m$ with positive integer $m$ is established as the sum of the effects of the original source and its $2 m-1$ images. This sum is replaced by a contour integral which is extended so as to obtain a formula valid for any positive $m$ and henceforth for any angle $\alpha$ of the wedge. Again, the solution is given by a contour integral. In more recent times a number of attempts have been made to apply integral transform methods $[20,17]$ and integral equation methods $[23,7,14,13]$ to the half plane diffraction problem. Finally, the problem of the diffraction by a perfectly conducting half plane of electromagnetic waves emitted by an arbitrarily oriented electric or magnetic dipole has also been solved [32, $39,15]$.

Sommerfeld's solution (plane waves incident on a half plane) is given in a form readily available for numerical computation inasmuch as it contains only the tabulated error function besides elementary functions. The solution of the other cases can either be given in the form of infinite series (usually suitable for the numerical evaluation of the near field) or in the form of integral expressions. A reduction of the latter to an asymptotic series has to be carried out if approximate expressions are desired for distances that are large compared to the wave length. Expressions of this form have been derived for the case of the diffraction of a plane wave by a wedge $[44,29]$. (An asymptotic expansion which fails to give the proper behavior in the neighborhood of the boundaries of geometric optics is ignored here [36].) The main feature of these asymptotic series is that their terms are generalized error functions. The work referred to above is concerned only with time harmonic problems.

Guest worker, National Bureau of Standards, from American University. Present address, Oregon State College, Corvallis, Ore. This research was carried out in part under Contract No. DA-11-022-0RD-2059 with the University of Wisconsin.

2 Figures in brackets indicate the literature references at the end of this paper. 
A second group of investigations centers around problems in which the dependence upon time is not periodic. This is the case of the diffraction of a pulse or a transient wave by a wedge or a half plane. This group of investigations was initiated by Sommerfeld [35]. The following contributions are listed here: Normal incidence of a plane pulse on a half plane [21,11]; twodimensional pulse incident on a half plane [12]; plane pulse (unit step function) incident on a wedge or a corner $[19,24,25]$; arbitrary pulse incident on a wedge or a corner [18]; cylindrical Dirac pulse incident on a half plane [38]; and spherical pulse (unit step function) incident on a half plane [40]. This paper will deal with various problems arising in the theory of the excitation of a perfectly reflecting wedge or corner by a plane, cylindrical, or spherical wave field.

The incident cylindrical wave is represented by an acoustic or electromagnetic line source parallel to the edge. The spherical wave is emitted by an acoustic point source or by a Hertz dipole with its axis parallel to the edge. The case of an incident plane wave field is obtained as the limiting case (for large distances of the source from the edge) of the cylindrical or spherical wave excitation.

In section 2 of this paper, various representations for the time harmonic solution of the problems outlined before are given with special emphasis to a representation upon which the investigations of sections 3 to 6 are based. A straightforward method is applied based upon the representation of the incident field in the form of a Kantorovich-Lebedev transform.

Section 3 contains a detailed discussion of the results obtained in section 2. Relations to geometric optics are discussed.

Section 4 contains an asymptotic expansion for the far field excited by the incidence of a plane wave on a wedge. This expansion is not of the Wiegrefe-Pauli type inasmuch as it consists of two error function terms and an asymptotic series with inverse powers of the variable. Remarkable is the fact, that this series represents asymptotically (for large distances from the edge) the deviation of the wedge field from the corresponding half-plane field.

Section 5 contains expressions for the energy radiated from a Hertz dipole in the presence of a perfectly reflecting wedge or corner (antenna and corner reflector).

Section 6 gives the pulse or transient solutions corresponding to the time harmonic problems investigated in section 2. It is shown that previously known results can be obtained as special cases of the expressions given here.

\section{Time Harmonic Wedge Diffraction Problems}

The case of the excitation of a perfectly reflecting wedge by a (acoustic or electromagnetic) line source parallel to the edge of the wedge is considered first. (The solution for the excitation by a point source can then be obtained by a further integration.) Cylindrical coordinates $(\rho, \phi, z)$ are introduced such that the $z$ axis coincides with the edge of the wedge and the walls of the wedge are bounded by $\phi=0$ and $\phi=\alpha$. Let the locations of the line source and the point of observation be characterized by $Q\left(\rho^{\prime}, \phi^{\prime}\right)$ and $P(\rho, \phi)$, respectively (fig. 1). Let the field inside the wedge be periodic"of the form

$$
\Phi=U(\rho, \phi) \exp (i \omega t),
$$

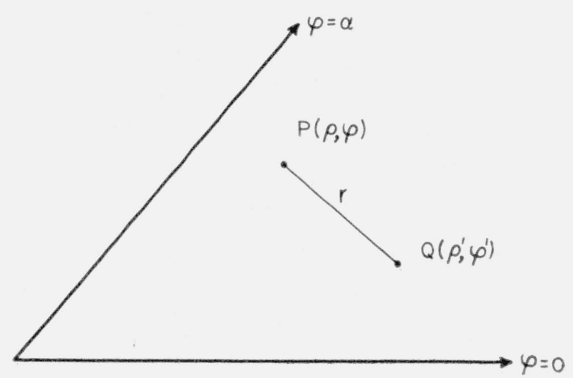

Figure 1. 
where $U$ satisfies the two-dimensional wave equation

$$
\Delta U+k^{2} U=\frac{\partial^{2} U}{\partial \rho^{2}}+\frac{1}{\rho} \frac{\partial U}{\partial \rho}+\frac{1}{\rho^{2}} \frac{\partial^{2} U}{\partial \phi^{2}}+k^{2} U=0 .
$$

A solution of (2) representing divergent waves is of the form

$$
U=\exp ( \pm i \nu \phi) H_{\nu}{ }^{(2)}(k \rho) .
$$

$H_{\nu}{ }^{(2)}(k \rho)$ is the second Hankel function, and $\nu$ is an arbitrary parameter. Following a procedure used elsewhere [26], the substitution

$$
k=2 \pi / \lambda=\omega / c=-i \gamma
$$

is introduced. This means that instead of (2) and (1)

$$
\Delta U-\gamma^{2} U=0, \quad \Phi=U(\rho, \phi) \exp (\gamma c t)
$$

is considered with a particular solution corresponding to (3)

where

$$
U=\exp ( \pm i \nu \phi) K_{\nu}(\gamma \rho),
$$

$$
K_{\nu}(z)=-\frac{1}{2} i \pi \exp \left(-i \frac{1}{2} \nu \pi\right) H_{\nu}{ }^{(2)}(-i z)
$$

denotes the modified Hankel function. The transition from $k$ to $\gamma$ amounts to the transition from a wave problem to an exponential decay problem. At first the exponential decay problem is solved, and the wave problem solution is established by returning from $\gamma$ to $k$. This will not only simplify questions with respect to convergence but the investigations in sections 4 and 6 are based upon the exponential decay solution and not on the time harmonic solution. The incident field in $P(\rho, \phi)$ due to the line source $Q\left(\rho^{\prime}, \phi^{\prime}\right)$ is given by

$$
U^{i}=H_{0}^{(2)}(k r)=\frac{2 i}{\pi} K_{0}\left\{\gamma\left[\rho^{2}+\rho^{\prime 2}-2 \rho \rho^{\prime} \cos \left(\phi-\phi^{\prime}\right)\right]^{1 / 2}\right\} .
$$

Let the total field $U$ be represented as a sum of the incident field $U^{i}$ and a reflected field $U^{r}$, where $U^{r}$ has no singularities within the wedge:

$$
U=U^{i}+U^{r} .
$$

In order to represent $U^{i}$ in a form suitable for the problem considered here it is advisable to express the modified Hankel function on the r. h. s. of (8) not by means of its well-known addition theorem [9, p. 44] but by the equivalent expression ((3A) appendix):

$$
\begin{aligned}
U^{i} & =\frac{2 i}{\pi} K_{0}\left\{\gamma\left[\rho^{2}+\rho^{\prime 2}-2 \rho \rho^{\prime} \cos \left(\phi-\phi^{\prime}\right)\right]^{1 / 2}\right\} \\
& =4 i \pi^{-2} \int_{0}^{\infty} K_{i \nu}(\gamma \rho) K_{i \nu}\left(\gamma \rho^{\prime}\right) \cosh \left[\nu\left(\pi-\left|\phi-\phi^{\prime}\right|\right)\right] d \nu,
\end{aligned}
$$

which is of the form (6). Let the reflected field be represented in the same form:

$$
U^{r}=4 i \pi^{-2} \int_{0}^{\infty} K_{i \nu}(\gamma \rho) K_{i \nu}\left(\gamma_{\rho^{\prime}}\right)\left[f_{1}(\nu) \exp (\nu \phi)+f_{2}(\nu) \exp (-\nu \phi)\right] d \nu
$$

The so far unknown functions $f_{1}$ and $f_{2}$ are to be determined by the boundary conditions. There are two cases of boundary conditions: (a) $U=0$ and (b) $\partial U / \partial \phi=0$ on the walls $\phi=0$ and $\phi=\alpha$ of the wedge. With electromagnetic waves on a perfect conducting wedge, 
case (a) refers to the electric field, case (b) refers to the magnetic field. With acoustic waves, case (a) refers to free wall, and case (b) refers to rigid wall.

This leads to

and

$$
\begin{aligned}
f_{1}+f_{2} & =-\cosh \left[\nu\left(\pi-\phi^{\prime}\right)\right] \\
f_{1} \exp (\nu \alpha)+f_{2} \exp (-\nu \alpha) & =-\cosh \left[\nu\left(\pi-\alpha+\phi^{\prime}\right)\right]
\end{aligned}
$$

$$
\begin{aligned}
f_{1}-f_{2} & =-\sinh \left[\nu\left(\pi-\phi^{\prime}\right)\right] \\
f_{1} \exp (\nu \alpha)-f_{2} \exp (-\nu \alpha) & =\sinh \left[\nu\left(\pi-\alpha+\phi^{\prime}\right)\right] .
\end{aligned}
$$

for cases (a) and (b), respectively. It may be noted that the boundary conditions have been satisfied by putting the integrand equal to zero. This can be justified by verifying that the solution thus obtained satisfies all necessary conditions [31]. But it may also be remarked that $U$ is represented as a Kontorovich-Lebedev transform [9, p. 75]. After $f_{1}$ and $f_{2}$ have been determined by (12) and (13) the solution is then, by (9), (10), and (11),

$$
U=4 i \pi^{-2} \int_{0}^{\infty} K_{i \nu}(\gamma \rho) K_{i \nu}\left(\gamma \rho^{\prime}\right) \frac{\sinh (\pi \nu)}{\sinh (\alpha \nu)}\left\{\cosh \left[\nu\left(\alpha-\left|\phi-\phi^{\prime}\right|\right)\right] \mp \cosh \left[\nu\left(\alpha-\phi-\phi^{\prime}\right)\right]\right\} d \nu .
$$

The upper and lower sign is valid for cases (a) and (b), respectively. The relation ( $(9 \mathrm{~A})$ appendix) shows that the convergence of the integral (14) at its upper limit for a real and positive $\gamma$ is secured when

$$
0 \leqq\left|\phi \mp \phi^{\prime}\right| \leqq 2 \alpha,
$$

and this region contains the whole region of the wedge. If one returns now to the wave problem replacing $\gamma$ by $i k$ according to (4) one arrives at the result

$U=-i \int_{0}^{\infty} \exp (\pi \nu) H_{i \nu}^{(2)}(k \rho) H_{i \nu}^{(2)}\left(k \rho^{\prime}\right) \frac{\sinh (\pi \nu)}{\sinh (\alpha \nu)}\left\{\cosh \left[\nu\left(\alpha-\left|\phi-\phi^{\prime}\right|\right)\right] \mp \cosh \left[\nu\left(\alpha-\phi-\phi^{\prime}\right)\right]\right\} d \nu$.

The behavior of the integrand at its upper limit leads by [9, p. 88, form. 22] to the condition

$$
\pi \leqq\left|\phi \mp \phi^{\prime}\right| \leqq 2 \alpha+\pi .
$$

This restricts the validity of (15) to the case of a wedge of an angle $\alpha$ larger than $\pi$ with the point of observation $P$ in the geometric shadow of the source $Q$. The expression (14) shall now be transformed into an infinite series. For this purpose the elementary relation [2, p. 368]

$$
\frac{\cosh [\nu(\alpha-\theta)]}{\sinh (\alpha \nu)}=\frac{\nu}{\alpha} \sum_{n=0}^{\infty} \frac{\epsilon_{n} \cos (n \pi \theta / \alpha)}{n^{2}+(n \pi / \alpha)^{2}}, \quad \epsilon_{0}=1, \quad \epsilon_{n}=2 \quad \text { for } n \geqq 1,
$$

valid for $0 \leqq \theta \leqq 2 \alpha$ is used. Term by term integration yields

$$
\int_{0}^{\infty} K_{i \nu}(\gamma \rho) K_{i \nu}\left(\gamma \rho^{\prime}\right) \frac{\cosh [\nu(\alpha-\theta)]}{\sinh (\nu \alpha)} \sinh (\pi \nu) d \nu=\frac{1}{\alpha} \sum_{n=0}^{\infty} \epsilon_{n} \cos (n \pi \theta / \alpha) \int_{0}^{\infty} \frac{\nu \sinh (\pi \nu)}{\nu^{2}+(n \pi / \alpha)^{2}} K_{i \nu}\left(\gamma \rho^{\prime}\right) K_{i \nu}(\gamma \rho) d v .
$$

The integral is known [10, vol. 2, p. 176]. (Note that this formula is not only valid for positive integers but for Re $n>0$ ). Hence

$$
\int_{0}^{\infty} \frac{\nu \sinh (\pi \nu)}{\nu^{2}+(n \pi / \alpha)^{2}} K_{i \nu}(\gamma \rho) K_{i \nu}\left(\gamma_{\rho}^{\prime}\right) d \nu=\frac{1}{2} \pi^{2} I_{n \pi / \alpha}(\gamma \rho) K_{n \pi / \alpha}\left(\gamma \rho^{\prime}\right)
$$

for $\rho\left\langle\rho^{\prime}\right.$ and for $\rho>\rho^{\prime}$ the same formula with $\rho$ and $\rho^{\prime}$ interchanged. Therefore, the field $U$ can be written as

$$
U=\frac{2 i}{\alpha} \sum_{n=0}^{\infty} \epsilon_{n}\left\{\cos \left[\frac{n \pi}{\alpha}(\phi-\phi)^{\prime}\right] \mp \cos \left[\frac{n \pi}{\alpha}(\phi+\phi)^{\prime}\right]\right\} I_{n \pi / \alpha}(\gamma \rho) K_{n \pi / \alpha}\left(\gamma \rho^{\prime}\right)
$$


for $\rho\left\langle\rho^{\prime}\right.$ and for $\left.\rho\right\rangle \rho^{\prime}$ the same formula with $\rho$ and $\rho^{\prime}$ interchanged. 'The expression (16) is valid for arbitrarily complex $\gamma$ and if $\gamma$ is replaced by $i k$ the solution for the corresponding wave problem becomes

$$
U=\frac{\pi}{\alpha} \sum_{n=0}^{\infty} \epsilon_{n}\left\{\cos \left[n \frac{\pi}{\alpha}\left(\phi-\phi^{\prime}\right)\right] \mp \cos \left[n \frac{\pi}{\alpha}\left(\phi+\phi^{\prime}\right)\right]\right\} J_{n \pi / \alpha}(k \rho) H_{n \pi / \alpha}^{(2)}\left(k \rho^{\prime}\right)
$$

for $\rho\left\langle\rho^{\prime}\right.$, and for $\left.\rho\right\rangle \rho^{\prime}$ the same expression is used with $\rho$ and $\rho^{\prime}$ interchanged. The preceding expressions (16) and (17) represent rapidly convergent series if the arguments of the Bessel functions are small. The expressions (16) and (17) shall now be transferred back into another integral expression which is the basis of the investigations in sections 3 and 6 . For this purpose the product $I_{n \pi / \alpha}(\gamma \rho) K_{n \pi / \alpha}\left(\gamma \rho^{\prime}\right)$ in (16) is replaced by an integral representation ((1A) appendix). This leads to the following expressions.

$$
\begin{aligned}
s & =\sum_{n=0}^{\infty} \epsilon_{n} \cos (n \pi \theta / \alpha) I_{n \pi / \alpha}(\gamma \rho) K_{n \pi / \alpha}\left(\gamma \rho^{\prime}\right) \\
& =\pi^{-1} \sum_{n=0}^{\infty} \epsilon_{n} \cos (n \pi \theta / \alpha) \int_{0}^{\pi} K_{0}\left[\gamma\left(\rho^{2}+\rho^{\prime 2}-2 \rho \rho^{\prime} \cos x\right)^{1 / 2}\right] \cos (n \pi x / \alpha) d x \\
& -\pi^{-1} \sum_{n=0}^{\infty} \epsilon_{n} \cos (n \pi \theta / \alpha) \sin \left(n \pi^{2} / \alpha\right) \int_{0}^{\infty} \exp (-n \pi x / \alpha) K_{0}\left[\gamma\left(\rho^{2}+\rho^{\prime 2}+2 \rho \rho^{\prime} \cosh x\right)^{1 / 2}\right] d x .
\end{aligned}
$$

The second sum vanishes when $\alpha=\pi / m, m=1,2,3, \ldots$ The two sums on the r. h. s. of (18) are denoted by $s_{1}$ and $s_{2}$.

Interchanging the order of summation and integration one finds

$$
\begin{gathered}
s_{1}=(2 \pi)^{-1} \lim _{N \rightarrow \infty} \int_{0}^{\pi} K_{0}\left[\gamma\left(\rho^{2}+\rho^{\prime 2}-2 \rho \rho^{\prime} \cos x\right)^{1 / 2}\right] \sum_{n=0}^{N} \epsilon_{n}\left\{\cos \left[n \frac{\pi}{\alpha}(x+\theta)\right]+\cos \left[n \frac{\pi}{\alpha}(x-\theta)\right]\right\} d x, \\
s_{2}=-\pi^{-1} \int_{0}^{\infty} K_{0}\left[\gamma\left(\rho^{2}+\rho^{\prime 2}+2 \rho \rho^{\prime} \cosh x\right)^{1 / 2}\right] \\
\cdot \sum_{n=1}^{\infty} \exp (-n \pi x / \alpha)\left\{\sin \left[n \frac{\pi}{\alpha}(\pi+\theta)\right]+\sin \left[n \frac{\pi}{\alpha}(\pi-\theta)\right]\right\} d x .
\end{gathered}
$$

The sum in the integrand of (20) can be summed [2, p. 203] and the result is

$$
\begin{aligned}
& s_{2}=-(2 \pi)^{-1} \int_{0}^{\infty} K_{0}\left[\gamma\left(\rho^{2}+\rho^{\prime 2}+2 \rho \rho^{\prime} \cosh x\right)^{1 / 2}\right] \\
& \left\{\frac{\sin \left[\frac{\pi}{\alpha}(\pi-\theta)\right]}{\cosh (\pi x / \alpha)-\cos \left[\frac{\pi}{\alpha}(\pi-\theta)\right]} \operatorname{sin[\frac {\pi }{\alpha }(\pi +\theta )]} \frac{\cosh (\pi x / \alpha)-\cos \left[\frac{\pi}{\alpha}(\pi+\theta)\right]}{d}\right\} d x .
\end{aligned}
$$

$s_{1}$ as given by (19) can be expressed as a sum of a finite number of terms. The result is (appendix)

$$
s_{1}=\alpha \pi^{-1} \sum_{r=r_{1}}^{r_{2}} K_{0}\left\{\gamma\left[\rho^{2}+\rho^{\prime 2}-2 \rho \rho^{\prime} \cos (2 \alpha r+\theta)\right]^{1 / 2}\right\} \text {. }
$$

The limits of the summation in $(22)$ are

$$
r_{1}=-\left[\frac{\pi+\theta}{2 \alpha}\right], \quad r_{2}=\left[\frac{\pi-\theta}{2 \alpha}\right]
$$

where the well-known symbol $\left[\frac{\pi \pm \theta}{2 \alpha}\right]$ means the largest positive or negative number which 
is smaller or equal to $\frac{\pi \pm \theta}{2 \alpha}$. The sum (22) is zero when $r_{1}>r_{2}$ and the first or last term of the sum has to be halved when $r_{1}$ or $r_{2}$ is an integer. With (18) to (23) the expression (16) for the field can also be written as

$$
U=F\left(\phi-\phi^{\prime}\right) \mp F\left(\phi+\phi^{\prime}\right),
$$

with

$$
\begin{gathered}
F(\theta)=2 i \pi^{-1} \sum_{r=r_{1}}^{r_{2}} K_{0}\left\{\gamma\left[\rho^{2}+\rho^{\prime 2}-\rho \rho^{\prime} \cos (2 \alpha r+\theta)\right]^{1 / 2}\right\}-i \alpha \pi^{-1} \int_{0}^{\infty} K_{0}\left[\gamma\left(\rho^{2}+\rho^{\prime 2}+2 \rho \rho^{\prime} \cosh x\right)^{1 / 2}\right] \\
\cdot\left\{\frac{\sin \left[\frac{\pi}{\alpha}(\pi-\theta)\right]}{\sin \left[\frac{\pi}{\alpha}(\pi+\theta)\right]}\right] d x, \frac{\cosh (\pi x / \alpha)-\cos \left[\frac{\pi}{\alpha}(\pi+\theta)\right]}{\cos (\pi)-\cos \left[\frac{\pi}{\alpha}(\pi-\theta)\right]}
\end{gathered}
$$

and $r_{1}$ and $r_{2}$ defined by (23).

\subsection{Spherical and Plane Field Excitation}

The formula [9, p. 95, formula 53]

$$
\left(r^{2}+z^{2}\right)^{-1 / 2} \exp \left[-\gamma\left(r^{2}+z^{2}\right)^{1 / 2}\right]=2 \pi^{-1} \int_{0}^{\infty} K_{0}\left[r\left(\gamma^{2}+v^{2}\right)^{1 / 2}\right] \cos (z v) d v,
$$

expresses the generation of a spherical wave function by means of a cylindrical wave function. To obtain the field $U$ corresponding to an incident spherical field multiply the r. h. s. of (8) and $(25)$ by $\cos (z v)$, replace $\gamma$ by $\left(\gamma^{2}+v^{2}\right)^{1 / 2}$ and integrate the resulting expression with respect to $v$ from 0 to $\infty$. This operation performed on (8) gives, using (26),

$$
2 i \pi^{-1} \int_{0}^{\infty} K_{0}\left[r\left(\gamma^{2}+v^{2}\right)^{1 / 2}\right] \cos (z v) d v=i\left(r^{2}+z^{2}\right)^{-\frac{1}{2}} \exp \left[-\gamma\left(r^{2}+z^{2}\right)^{1 / 2}\right]
$$

i. e., an incident spherical field originating from a source point $Q\left(\rho^{\prime}, \phi^{\prime}, 0\right)$. The same operation performed on (25) gives similar expressions. (For similar procedures, see [42, 5]). It follows then that in the case of an incident plane, cylindrical or spherical field the field $U$ within the wedge of an angle $\alpha$ can be written in the form

$$
U=F\left(\phi-\phi^{\prime}\right) \mp F\left(\phi+\phi^{\prime}\right) .
$$

$F(\theta)$ is expressed as the sum of two functions $F_{1}$ and $F_{2}$ :

$$
F(\theta)=F_{1}(\theta)+F_{2}(\theta),
$$

where

$$
\begin{gathered}
F_{1}(\theta)=\sum_{r=r_{1}}^{r_{2}} f(\theta+2 \alpha r), \\
F_{2}(\theta)=-(2 \alpha)^{-1} \int_{0}^{\infty} H(x)\left\{\frac{\sin \left[\frac{\pi}{\alpha}(\pi-\theta)\right]}{\sin \left[\frac{\pi}{\alpha}(\pi+\theta)\right]} \operatorname{cosh(\pi x/\alpha )-\operatorname {cos}[\frac {\pi }{\alpha }(\pi -\theta )]^{+}\operatorname {cosh}(\pi x/\alpha )-\operatorname {cos}[\frac {\pi }{\alpha }(\pi +\theta )]}\right\} d x .
\end{gathered}
$$

The representations (28) are also valid for complex values of $\gamma$ with $\operatorname{Re} \gamma \geqq 0$ and consequently for $\gamma=i k$ with $\operatorname{Im} k \leqq 0$. The functions $f$ and $H$ in (30) and (31) depend on the character of the excitation and are listed below. (The incident plane field case is obtained as the limiting case of the incident spherical field case for $\rho^{\prime} \rightarrow \infty$.) 


\begin{tabular}{|c|c|}
\hline $\begin{array}{c}f\left(\phi-\phi^{\prime}\right) \\
(\text { incident field })\end{array}$ & $H(x)$ \\
\hline $\begin{array}{c}\exp \left[\gamma \rho \cos \left(\phi-\phi^{\prime}\right)\right] \\
(\text { plane field })\end{array}$ & $\exp (-\gamma \rho \cosh x)$ \\
\hline $2 i \pi^{-1} K_{0}(\gamma r)$ & $2 i \pi^{-1} K_{0}\left[\gamma\left(\rho^{2}+\rho^{\prime 2}+2 \rho \rho^{\prime} \cosh x\right)^{1 / 2}\right]$ \\
$(\operatorname{cylindrical~field~})$ & $\left(\rho^{2}+\rho^{\prime 2}+z^{2}+2 \rho \rho^{\prime} \cosh x\right)^{-1 / 2}$ \\
\hline$\left(r^{2}+z^{2}\right)^{-1 / 2} \exp \left[-\gamma\left(r^{2}+z^{2}\right)^{1 / 2}\right]$ & $\cdot \exp \left[-\gamma\left(\rho^{2}+\rho^{\prime 2}+z^{2}+2 \rho \rho^{\prime} \cosh x\right)^{1 / 2}\right]$. \\
\hline$($ spherical field $)$ & \\
\hline
\end{tabular}

Functions $f$ and $H$ for the wave problem

\begin{tabular}{|c|c|}
\hline $\begin{array}{c}f\left(\phi-\phi^{\prime}\right) \\
(\text { incident field })\end{array}$ & $H(x)$ \\
\hline $\begin{array}{c}\exp \left[i k \rho \cos \left(\phi-\phi^{\prime}\right)\right] \\
(\text { plane wave })\end{array}$ & $\exp (-i k \rho \cosh x)$ \\
\hline $\begin{array}{c}H_{0}^{(2)}(k r) \\
(\text { cylindrical wave })\end{array}$ & $H_{0}^{(2)}\left[k\left(\rho^{2}+\rho^{\prime 2}+2 \rho \rho^{\prime} \cosh x\right)^{1 / 2}\right]$ \\
\hline$\left(r^{2}+z^{2}\right)^{-1 / 2} \exp \left[-i k\left(r^{2}+z^{2}\right)^{1 / 2}\right]$ & $\left(\rho^{2}+\rho^{\prime 2}+z^{2}+2 \rho \rho^{\prime} \cosh x\right)^{-1 / 2}$ \\
$($ spherical wave $)$ & $\cdot \exp \left[-i k\left(\rho^{2}+\rho^{\prime 2}+2 \rho \rho^{\prime} \cosh x+z^{2}\right)^{1 / 2}\right]$. \\
\hline
\end{tabular}

$$
r=\left[\rho^{2}+\rho^{\prime 2}-2 \rho \rho^{\prime} \cos \left(\phi-\phi^{\prime}\right)\right]^{1 / 2} .
$$

The function $f\left(\phi-\phi^{\prime}\right)$ represents the analytic character of the incident plane, cylindrical, or spherical field. The upper or lower sign in (28) has to be taken according as an incident electromagnetic or acoustic field is considered. The total field $U$ is now represented in three different forms, (14), (16), (28) and (15), (17), (28) for the exponential decay and the wave problem, respectively. Since in the following, (28) will be dealt with exclusively, the expressions for $U$ of the form (14), (16), (15), and (17) corresponding to an incident plane or spherical field are not listed here. They are obtained in the same manner. The field as represented by (28) consists of the sum of two functions $F_{1}$ and $F_{2}$. Each term $r=0$ of $F_{1}$ represents a field of the same character as the incident field $(r=0)$ but originating from one of the images of the source $Q$ with respect to the walls of the wedge. The number of the images and their location depend on $r_{1}$ and $r_{2}$ and henceforth on the configuration. $F_{2}$ is given by an integral expression which vanishes when the angle of the wedge is of the form $\pi / m, \quad m=1,2,3$, . . . It is easily seen that in this case $U=F_{1}\left(\phi-\phi^{\prime}\right) \pm F_{1}\left(\phi \mp \phi^{\prime}\right)$ is composed of the effect of the original source and its $2 m-1$ images. In case of an incident plane field and an angle $2 \pi$ (half plane), $F_{2}$ can be reduced to the error integral (39).

\section{Discussion of $F_{1}$ and $F_{2}$ for Particular Cases}

It is obvious that the number of terms of the sum (30) can be made arbitrarily large if $\alpha$ is arbitrarily small. The case $0<\alpha<\pi$ shall from now on be referred to as a corner. It will be shown now that for a wedge $(\pi<\alpha<2 \pi)$ the sum (30) consists of at most one term. Two cases must be distinguished here: case a, $\alpha>\pi, 0<\phi^{\prime}\langle\alpha-\pi$, figure 2 ; and case $\mathrm{b}, \alpha\rangle \pi$, $\alpha-\pi<\phi^{\prime}<\frac{1}{2} \alpha$, figure 3 . The restriction $\phi<\frac{1}{2} \alpha$ means no loss of generality. 


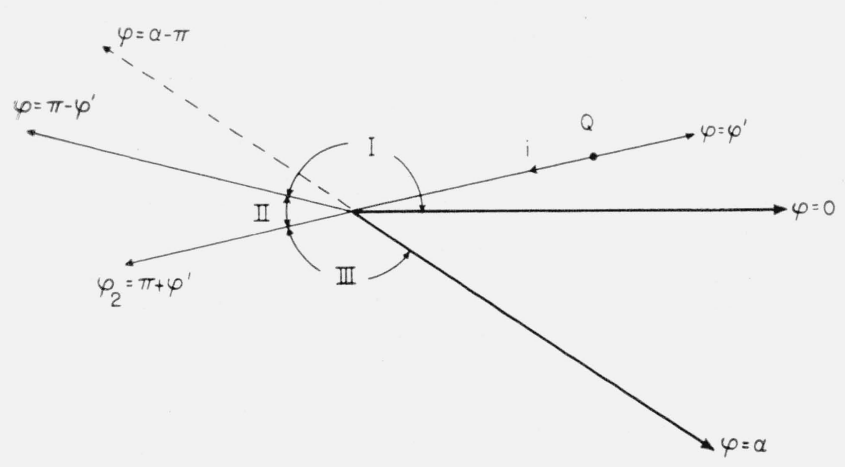

Figure 2.

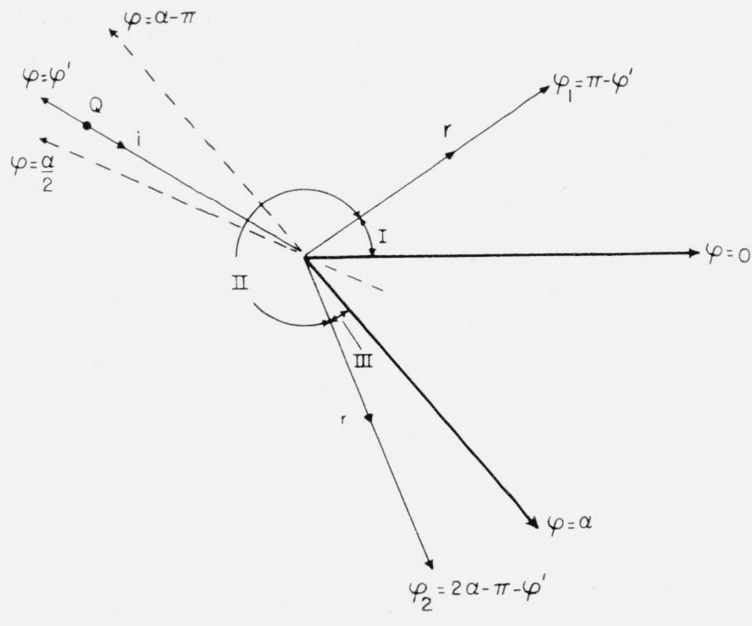

Figure 3.

These cases represent the two possible configurations.

Case $a$. The direction $\phi^{\prime}$ of the source $?$ is such, that a geometric shadow region exists. The boundary of the geometric reflection is $\phi_{1}=\pi-\phi^{\prime}$ and the boundary of the geometric shadow is $\phi_{2}=\pi+\phi^{\prime}$.

Case $b$. 'The direction $\phi^{\prime}$ of the source $?$ is such that no geometric shadow region exists. The boundaries of the geometric reflection with respect to the walls $\phi=0$ and $\phi=\alpha$ are $\phi_{1}=\pi-\phi^{\prime}$ and $\phi_{2}=2 \alpha-\pi-\alpha^{\prime}$, respectively.

Let the region $0 \leqq \phi \leqq \alpha$ be subdivided into three regions I, II, III bounded by the walls and the boundaries of geometric opties $\phi=\phi_{1}$ and $\phi=\phi_{2}$. They are defined by:

and

Region I. $\quad 0 \leqq \phi \leqq \phi_{1}$

Region II. $\quad \phi_{1} \leqq \phi \leqq \phi_{2}$

Region III. $\quad \phi_{2} \leqq \phi \leqq \alpha$

$$
\begin{gathered}
\phi_{1}=\pi-\phi^{\prime}, \quad \phi_{2}=\pi+\phi^{\prime} \quad \text { case a, } \\
\phi_{1}=\pi-\phi^{\prime}, \quad \phi_{2}=2 \alpha-\pi-\phi^{\prime} \quad \text { case b. }
\end{gathered}
$$

In these cases the limits $r_{1}, r_{2}$ of (30) are easily found to be as listed below.

\begin{tabular}{|c|c|c|c|c|c|c|}
\hline \multicolumn{5}{|c|}{ Case a } & \multicolumn{3}{c|}{ Case b } \\
\hline Region & I & II & III & I & II & III \\
\hline$r_{1}, r_{2}$ & 0,0 & 0,0 & $0,-1$ & 0,0 & 0,0 & 0,0 \\
\hline$r_{1}, r_{2}$ & 0,0 & $0,-1$ & $0,-1$ & 0,0 & $0,-1$ & $-1,-1$ \\
\hline
\end{tabular}

The upper or lower line for $\left(r_{1}, r_{2}\right)$ is valid according as $\theta=\phi-\phi^{\prime}$ or $\theta=\phi+\phi^{\prime}$. With these results one obtains by (30) for both cases a and b,

$$
\begin{array}{ll}
F_{1}\left(\phi-\phi^{\prime}\right) \mp F_{1}\left(\phi+\phi^{\prime}\right)=f\left(\phi-\phi^{\prime}\right) \mp f\left(\phi+\phi^{\prime}\right) & \text { valid in I, } \\
F_{1}\left(\phi-\phi^{\prime}\right) \mp F_{1}\left(\phi+\phi^{\prime}\right)=f\left(\phi-\phi^{\prime}\right) & \text { valid in II, }
\end{array}
$$


while in region III

$$
\begin{array}{cc}
F_{1}\left(\phi-\phi^{\prime}\right) \mp F_{1}\left(\phi+\phi^{\prime}\right)=0 & \text { case a } \\
F_{1}\left(\phi-\phi^{\prime}\right) \mp F_{1}\left(\phi+\phi^{\prime}\right)=f\left(\phi-\phi^{\prime}\right) \mp f\left(\phi+\phi^{\prime}-2 \alpha\right) & \text { case b. }
\end{array}
$$

Equations (35) to (38) together with (31) give the representation for the field $U$ by (28) and (29) in agreement with the results obtained elsewhere [26]. The case of a source within a corner $(0<\alpha<\pi)$ (fig. 4) and referred to as case $\mathrm{c}$ is treated the same way. At first $r_{1}$ and $r_{2}$ are determined by $(23)$ and then $F_{1}$ by (30). Together with $F_{2}$ by $(31)$, the field $U$ is known by $(28)$.

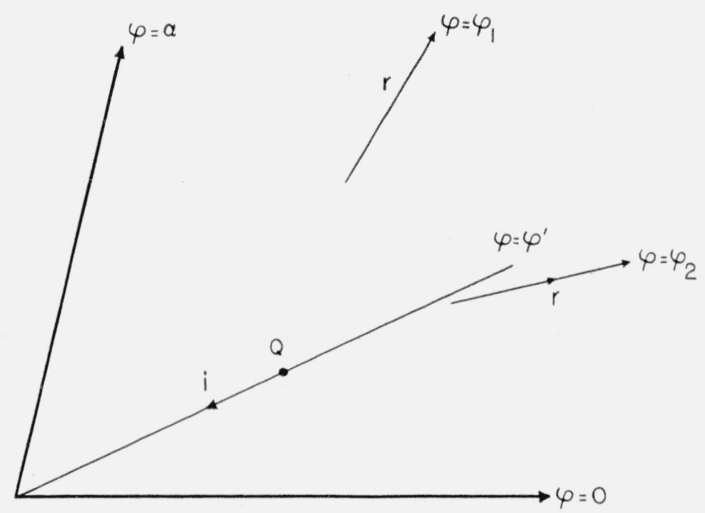

FIGURE 4 .

Analogous to the cases a and b there exist two directions $\phi_{1}$ and $\phi_{2}$ in which a plane wave field incident on the corner emerges after multiple reflections on the walls. They are given in (50) and (51).

The second function $F_{2}(\theta)$ given by the integral (31) can be given explicitly when $\alpha=2 \pi$ (half plane) and when the incident field is a plane field. Since this result will be needed later it is represented again although known [34]. Now, for a plane incident field, $f\left(\phi-\phi^{\prime}\right)$ $=\exp \left[\gamma \rho \cos \left(\phi-\phi^{\prime}\right)\right]$ and $H(x)=\exp (-\gamma \rho \cosh x)$. For $\alpha=2 \pi$,

$$
F_{2}(\theta)=-\pi^{-1} \cos \left(\frac{1}{2} \theta\right) \int_{0}^{\infty} \cosh \left(\frac{1}{2} x\right)(\cosh x+\cos \theta)^{-1} \exp (-\gamma \rho \cosh x) d x,
$$

by (31). The substitution $\cosh x=1+t$ gives

$$
F_{2}(\theta)=-\pi^{-1} 2^{-1 / 2} \cos \left(\frac{1}{2} \theta\right) \exp (-\gamma \rho) \int_{0}^{\infty} t^{-1 / 2}(1+\cos \theta+t)^{-1} \exp (-\gamma \rho t) d t .
$$

This integral is known [10, vol. 1, p. 136]. Thus

where

$$
F_{2}(\theta)=-\frac{1}{2} \cos \left(\frac{1}{2} \theta\right)\left|\sec \left(\frac{1}{2} \theta\right)\right| \operatorname{Erfc}\left[(2 \gamma \rho)^{1 / 2}\left|\cos \frac{1}{2} \theta\right|\right] \exp (\gamma \rho \cos \theta),
$$

$$
\operatorname{Erfc}(z)=2 \pi^{-1 / 2} \int_{z}^{\infty} \exp \left(-t^{2}\right) d t
$$

Since the half plane case is represented by case a, as described in figure 1, the matching expression for $F_{1}$ in the three regions is known by (35) to (37), and the field $U(28)$ excited by an incident plane field on a perfectly reflecting half plane can be written down explicitly. It can easily be verified that $U$ can be represented by an expression which is valid over the whole range of the half plane observing that by (40)

$$
\operatorname{Erf}(-z)=2-\operatorname{Erfc}(z),
$$


and so $U$ becomes

$U=\exp \left[\gamma \rho \cos \left(\phi-\phi^{\prime}\right)\right]\left\{1-\frac{1}{2} \operatorname{Erfc}\left[(2 \gamma \rho)^{1 / 2} \cos \left(\frac{1}{2} \phi-\frac{1}{2} \phi^{\prime}\right)\right]\right\}$

$$
\mp \exp \left[\gamma \rho \cos \left(\phi+\phi^{\prime}\right)\right]\left\{1-\frac{1}{2} \operatorname{Erfc}\left[(2 \gamma \rho)^{1 / 2} \cos \left(\frac{1}{2} \phi+\frac{1}{2} \phi^{\prime}\right)\right]\right\},
$$

for the exponential decay problem. The solution for the wave problem is obtained as $U=\exp \left[i k \rho \cos \left(\phi-\phi^{\prime}\right)\right]\left\{1-\frac{1}{2} \operatorname{Erfc}\left[(2 i k \rho)^{1 / 2} \cos \left(\frac{1}{2} \phi-\frac{1}{2} \phi^{\prime}\right)\right]\right\}$

$$
\mp \exp \left[i k \rho \cos \left(\phi+\phi^{\prime}\right)\right]\left\{1-\frac{1}{2} \operatorname{Erfc}\left[(2 i k \rho)^{1 / 2} \cos \left(\frac{1}{2} \phi+\frac{1}{2} \phi^{\prime}\right)\right]\right\} \cdot
$$

(For tables of the error function for complex argument see [6].)

In general case, $F_{2}$ consists of a sum of two integrals of the form

with

$$
F_{2}(\theta)=I(\alpha, \pi-\theta)+I(\alpha, \pi+\theta),
$$

$$
I(\alpha, \psi)=-(2 \alpha)^{-1} \int_{0}^{\infty} H(x) \sin (\pi \psi / \alpha)\left[(\cosh (\pi x / \alpha)-\cos (\pi \psi / \alpha)]^{-1} d x\right.
$$

Obviously the denominator of the integrand (45) is zero at its lower limit $x=0$ when

$$
\psi=\psi_{n}=\pi \pm\left(\phi \pm \phi^{\prime}\right)=2 n \alpha, \quad n=0, \pm 1, \pm 2, \ldots .
$$

But the numerator of the integrand vanishes likewise for the same value. It is easily shown [27] that

$$
\lim _{\psi \rightarrow \pm 0} I(\alpha, \psi)=\mp \frac{1}{2} H(0)
$$

and the behavior of (45) is the same for $\psi \rightarrow \pm 2 n \alpha$. It may be noted that (47) does not depend on the angle of the wedge. For any configuration is always

$$
0<\phi+\phi^{\prime}<2 \alpha, \quad 0<\left|\phi-\phi^{\prime}\right|<\alpha .
$$

This condition restricts (46) to only two possible directions $\phi_{1}$ and $\phi_{2}$ which define the boundaries of geometric optics as outlined before. They are defined by

$$
\text { case a: } \phi_{1}=\pi-\phi^{\prime},(n=0) ; \quad \phi_{2}=\pi+\phi^{\prime},(n=0),
$$

case b: $\quad \phi_{1}=\pi-\phi^{\prime},(n=0) ; \quad \phi_{2}=2 \alpha-\pi-\phi^{\prime},(n=1)$,

in agreement with (36). A choice of values for the parameter $n$ other than given above is incompatible with the configurations under consideration. In order to treat case $c$ it is practical to subdivide this case according as the angle $\alpha$ of the corner can be described as

$$
\begin{aligned}
& \alpha=\pi /(2 l+\epsilon), \quad l=1,2,3, \ldots \quad{\text { case } c_{1}} \\
& \alpha=\pi /(2 l+1+\epsilon), l=0,1,2, \ldots \quad \text { case } c_{2},
\end{aligned}
$$

where $\epsilon$ is a number between 0 and 1 . Then the relation (46) leads to

and

$$
\left.\begin{array}{l}
\phi_{1}=\left|\epsilon \alpha-\phi^{\prime}\right| \\
\phi_{2}=\operatorname{Min}\left[\alpha(2-\epsilon)-\phi^{\prime}, \phi^{\prime}+\epsilon \alpha\right]
\end{array}\right\} \text { case } c_{1}
$$

$$
\left.\begin{array}{l}
\phi_{1}=\left|\alpha(1-\boldsymbol{\epsilon})-\phi^{\prime}\right| \\
\phi_{2}=\operatorname{Min}\left[\alpha(1+\boldsymbol{\epsilon})-\phi^{\prime}, \alpha(1-\boldsymbol{\epsilon})+\phi^{\prime}\right]
\end{array}\right\} \text { case } c_{2}
$$


and $\epsilon$ is connected with $\alpha$ by (49). In case $\epsilon=0$, this leads to $\varphi_{1}=\varphi_{2}$ and particularly,

$$
\begin{gathered}
\phi_{1}=\phi_{2}=\phi^{\prime} \quad \text { when } \alpha=\pi /(2 l) \\
\phi_{1}=\phi_{2}=\alpha-\phi^{\prime} \quad \text { when } \alpha=\pi /(2 l+1) .
\end{gathered}
$$

An elementary expression for the upper limit of $I(\alpha, \psi)$ can be given. Integration of (45) by parts gives

$-\pi I\left(\alpha_{1} \psi\right)=\left.H(x) \arctan \left[\operatorname{cotan}\left(\frac{1}{2} \pi \psi / \alpha\right) \tanh \left(\frac{1}{2} \pi x / \alpha\right)\right]\right|_{0} ^{\infty}$

$$
-\int_{0}^{\infty} H^{\prime}(x) \arctan \left[\operatorname{cotan}\left(\frac{1}{2} \pi \psi / \alpha\right) \tanh \left(\frac{1}{2} \pi x / \alpha\right)\right] d x
$$

Since, with the exception of the plane wave case, $H(x) \rightarrow 0$ when $x \rightarrow \infty$ it becomes

and hence

$$
I\left(\alpha_{1} \psi\right)=\pi^{-1} \int_{0}^{\infty} H^{\prime}(x) \arctan \left[\operatorname{cotan}\left(\frac{1}{2} \pi \psi / \alpha\right) \tanh \left(\frac{1}{2} \pi x / \alpha\right)\right] d x,
$$

$$
\left|I\left(\alpha_{1} \psi\right)\right| \leqq \pi^{-1} \int_{0}^{\infty}\left|H^{\prime}(x)\right|\left|\arctan \left[\operatorname{cotan}\left(\frac{1}{2} \pi \psi / \alpha\right) \tanh \left(\frac{1}{2} \pi x / \alpha\right)\right]\right| d x
$$

and, after replacing $\tanh \left(\frac{1}{2} \pi x / \alpha\right)$ by its upper limit 1 ,

$$
\left|I\left(\alpha_{1} \psi\right)\right| \leqq|H(0)|\left|\arctan \left[\operatorname{cotan}\left(\frac{1}{2} \pi \psi / \alpha\right)\right]\right| \pi^{-1} .
$$

The equal sign holds when $\psi$ has one of the values defined by (46) and the accuracy of the inequality (53) increases with decreasing $\alpha$. Since, in general $\frac{1}{2} \pi \psi / \alpha$ will not lie between 0 and $\pi$ the "reduced" property

$$
\frac{1}{2} \pi \bar{\psi} / \alpha=\frac{1}{2} \pi \psi / \alpha \pm \pi l_{n}, \quad l_{n}=0,1,2, \ldots,
$$

is introduced and $l_{n}$ is such that $\frac{1}{2} \pi \bar{\psi} / \alpha$ is between 0 and $\pi$. Thus

and finally

$$
\left|I\left(\alpha_{1} \psi\right)\right| \leqq \pi^{-1}|H(0)|\left|\arctan \left[\operatorname{cotan}\left(\frac{1}{2} \pi \bar{\psi} / \alpha\right)\right]\right|,
$$

$$
\left|I\left(\alpha_{1} \psi\right)\right| \leqq \frac{1}{2}|H(0)||1-\bar{\psi} / \alpha| .
$$

\section{Asymptotic Expansions}

The derivation of an asymptotic expansion of the field $U$ for large distances from the edge of the wedge is equivalent with the reduction of the integral (31) to an asymptotic series. Comparatively simple is the case of a plane field incidence (fig. 5). Then for the exponential decay problem

$$
H(x)=\exp (-\gamma \rho \cosh x) .
$$

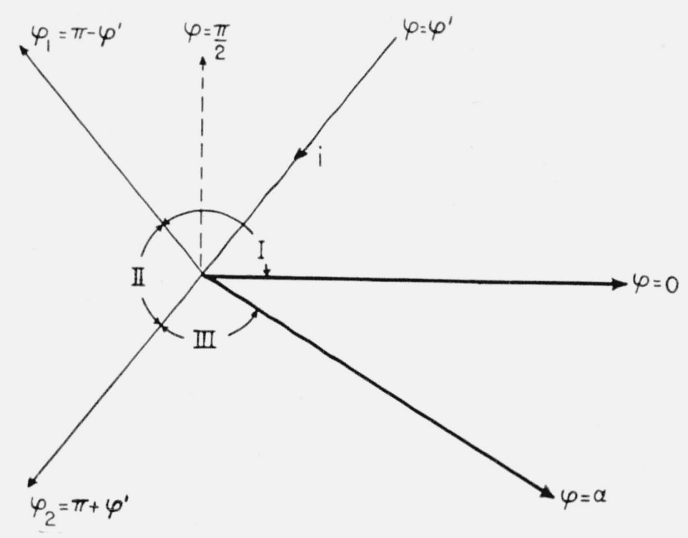

Figurea 5 . 
In this case $F_{2}(\theta)$ is represented as a Laplace transform with $\gamma \rho$ as parameter. Methods for the asymptotic development of integrals of this type are known [8, p. 45]. Since by (44) $F_{2}(\theta)$ is composed of two integrals of the form (45) it is sufficient to investigate

$$
I(\alpha, \psi)=-(2 \alpha)^{-1} \sin (\pi \psi / \alpha) \int_{0}^{\infty}[\cosh (\pi x / \alpha)-\cos (\pi \psi / \alpha)]^{-1} \exp (-\gamma \rho \cosh x) d x,
$$

and $\psi$ has one of the values $\pi \pm\left(\phi \pm \phi^{\prime}\right)$. The substitution

transforms $(57)$ into

$$
\cosh x=1+t
$$

$$
I(\alpha, \psi)=-\exp (-\gamma \rho) \int_{0}^{\infty} t^{-1 / 2} K(t) \exp (-\gamma \rho t) d t
$$

with

$$
K(t)=(2 \alpha)^{-1} \sin (\pi \psi / \alpha)(2+t)^{-1 / 2}\left\{\cosh \left[\pi / \alpha \log \left(1+t+\left(t^{2}+2 t\right)^{\frac{1}{2}}\right]-\cosh (\pi \psi / \alpha)\right\}^{-1} .\right.
$$

Thus (59) is represented as a Laplace transform. For the following, the discussion shall be restricted to case a, as described in section 3 and fig. 2. Cases b and c, as described in secticn 3, can be treated in the same manner. Now, $K(t)$ as given by $(60)$ represents a regular function in the vicinity of $t=0$ provided that $\phi \pm \phi^{\prime} \neq \pi$ (corresponding to $\psi \neq 0$ by (46)). The singularities of $K(t)$ lie on the negative axis and the smallest of their moduli is equal to $\operatorname{Min}\left[1+\cos \left(\phi \pm \phi^{\prime}\right)\right]$.

The Taylor expansion of $K(t)$ near the origin is

$$
K(t)=\sum_{n=0}^{\infty} A_{n}\left(\alpha_{1} \psi\right) t^{n}, \quad|t|<\operatorname{Min}\left[1+\cos \left(\phi \pm \phi^{\prime}\right)\right],
$$

with

$$
A_{n}\left(\alpha_{1} \psi\right)=(n !)^{-1}\left[d^{n} K(t) /\left(d t^{n}\right)\right]_{t=0} .
$$

The first two coefficients are

$$
\begin{gathered}
A_{0}\left(\alpha_{1} \psi\right)=(2 \alpha)^{-1} 2^{-1 / 2} \operatorname{cotan}\left(\frac{1}{2} \pi \psi / \alpha\right) \\
A_{1}(\alpha, \psi)=-(8 \alpha)^{-1} 2^{-1 / 2} \operatorname{cotan}\left(\frac{1}{2} \pi \psi / \alpha\right)\left\{1+2(\pi / \alpha)^{2}\left[\sin \left(\frac{1}{2} \pi \psi / \alpha\right)\right]^{-2}\right\}
\end{gathered}
$$

The asymptotic expansion of $I\left(\alpha_{1} \psi\right)$ for large $\gamma \rho$ is obtained by inserting the series (61) into (59) and integrating term by term [8, p. 48, theorem 6]. The result is

$$
I(\alpha, \psi) \sim-\exp (-\gamma \rho) \sum_{n=0}^{\infty} A_{n}\left(\alpha_{1} \psi\right) \Gamma\left(n+\frac{1}{2}\right)(\gamma \rho)^{-n-1 / 2}, \quad-3 \pi / 2<\arg \gamma<3 \pi / 2
$$

The coefficients $A_{n}$ tend to infinity when $\phi \pm \phi^{\prime}$ tends to $\pi$ (and hence $\psi$ to 0 ) and the expansion (64) becomes useless. This is the case when the configuration is such that one of the boundaries of geometric optics is approached. In this case the point $t=0$ becomes a singular point of $K(t)$ and no expansion of the type (61) exists. In order to obtain an asymptotic expansion which is valid over the whole region one proceeds as follows. The integrand of (57) at $x=0$ is equal to $-(2 \alpha)^{-1} \operatorname{cotan}\left(\frac{1}{2} \pi \psi / \alpha\right)$. In the vicinity of $\psi=0$ (the boundaries of geometric optics)

$$
-(2 \alpha)^{-1} \operatorname{cotan}\left(\frac{1}{2} \pi \psi / \alpha\right)=-(\pi \psi)^{-1}+O(\psi)
$$

and the singular part of (65) is independent of the angle $\alpha$. Let the field $U$ be now written in the form

$$
U=(U)_{\alpha=2 \pi}+\left[U-(U)_{\alpha=2 \pi}\right]
$$

and by (28), (29), and (35) to (37) 


$$
U=(U)_{\alpha=2 \pi}+F_{2}\left(\phi-\phi^{\prime}\right)-\left[F_{2}\left(\phi--\phi^{\prime}\right)\right]_{\alpha=2 \pi} \mp\left\{F_{2}\left(\phi+\phi^{\prime}\right)-\left[F_{2}\left(\phi+\phi^{\prime}\right)\right]_{\alpha=2 \pi}\right\} .
$$

Now, $(U)_{\alpha=2 \pi}$ is given by (42), and by (44)

$$
F_{2}\left(\phi \pm \phi^{\prime}\right)-\left[F_{2}\left(\phi \pm \phi^{\prime}\right)\right]_{\alpha=2 \pi}=I\left(\alpha, \pi-\phi \mp \phi^{\prime}\right)-I\left(2 \pi, \pi-\phi \mp \phi^{\prime}\right)+I\left(\alpha, \pi+\phi \pm \phi^{\prime}\right)-\left(I\left(2 \pi, \pi+\phi \pm \phi^{\prime}\right) .\right.
$$

The difference

$$
\begin{aligned}
& I\left(\alpha, \pi-\phi \mp \phi^{\prime}\right)-I\left(2 \pi, \pi-\phi \mp \phi^{\prime}\right) \\
& =-\int_{0}^{\infty}\left[\frac{(2 \alpha)^{-1} \sin \left[\frac{\pi}{\alpha}\left(\pi-\phi \mp \phi^{\prime}\right)\right]}{\cosh (\pi x / \alpha)-\cos \left[\frac{\pi}{\alpha}\left(\pi-\phi \mp \phi^{\prime}\right)\right]}-\frac{(4 \pi)^{-1} \cos \left(\frac{1}{2} \phi \pm \frac{1}{2} \phi^{\prime}\right)}{\cosh \left(\frac{1}{2} x\right)-\sin \left(\frac{1}{2} \phi \pm \frac{1}{2} \phi^{\prime}\right)}\right] \exp \left(-\gamma_{\rho} \cosh x\right) d x
\end{aligned}
$$

is by (65) such that the integrand of (68) vanishes identically when $\phi \pm \phi^{\prime}=\pi$, and the asymptotic expansion of (68) is given as the difference of two series of the type (64) whose coefficients $A_{n}\left(\alpha, \pi-\phi \mp \phi^{\prime}\right)-A_{n}\left(2 \pi, \pi-\phi \mp \phi^{\prime}\right)$ tend to zero when $\phi \mp \phi^{\prime}$ tends to $\pi$. Using (64) one arrives at the asymptotic expansion

$$
\begin{aligned}
F_{2}\left(\phi-\phi^{\prime}\right)-\left[F_{2}\left(\phi-\phi^{\prime}\right)\right]_{\alpha=2 \pi} \pm\left\{F_{2}\left(\phi+\phi^{\prime}\right)-\left[F_{2}\left(\phi+\phi^{\prime}\right)\right]_{\alpha=2 \pi}\right\}=S(\gamma \rho) & \\
& \sim-\exp (-\gamma \rho) \sum_{n=0}^{\infty} B_{n}\left(\gamma_{\rho}\right)^{-n-1 / 2}, \quad-3 \pi / 2<\arg \gamma<3 \pi / 2 .
\end{aligned}
$$

The coefficients $B_{n}$ are defined by

$$
\begin{array}{r}
B_{n}=\Gamma\left(n+\frac{1}{2}\right)\left\{A_{n}\left(\alpha, \pi-\phi+\phi^{\prime}\right)-A_{n}\left(2 \pi, \pi-\phi+\phi^{\prime}\right)+A_{n}\left(\alpha, \pi+\phi-\phi^{\prime}\right)-A_{n}\left(2 \pi, \pi+\phi-\phi^{\prime}\right)\right. \\
\left.\mp\left[A_{n}\left(\alpha, \pi-\phi-\phi^{\prime}\right)-A_{n}\left(2 \pi, \pi-\phi-\phi^{\prime}\right)+A_{n}\left(\alpha, \pi+\phi+\phi^{\prime}\right)-A_{n}\left(2 \pi, \pi+\phi+\phi^{\prime}\right)\right]\right\}
\end{array}
$$

With the $A_{n}$ given by (62), (63). It follows then from (67), (42), and (69) for the field excited by an incident plane field on a perfectly reflecting wedge of an angle $\alpha$ for case a (fig. 5)

$U=\exp \left[\gamma \rho \cos \left(\phi-\phi^{\prime}\right)\right]\left\{1-\frac{1}{2} \operatorname{Erfc}\left[(2 \gamma \rho)^{1 / 2} \cos \left(\frac{1}{2} \phi-\frac{1}{2} \phi^{\prime}\right)\right]\right\}$

$$
\mp \exp [\gamma \rho \cos (\phi+\phi)]\left\{1-\frac{1}{2} \operatorname{Erfc}\left[(2 \gamma \rho)^{1 / 2} \cos \left(\frac{1}{2} \phi+\frac{1}{2} \phi^{\prime}\right)\right]\right\}+S(\gamma \rho),
$$

where $S(\gamma \rho)$ is asymptotically given by (69). It follows then for the wave problem $U=\exp \left[i k \rho \cos \left(\phi-\phi^{\prime}\right)\right]\left\{1-\frac{1}{2} \operatorname{Erfc}\left[(2 i k \rho)^{1 / 2} \cos \left(\frac{1}{2} \phi-\frac{1}{2} \phi^{\prime}\right)\right]\right\}$

$$
\mp \exp \left[i k \rho \cos \left(\phi+\phi^{\prime}\right)\right]\left\{1-\frac{1}{2} \operatorname{Erfc}\left[(2 i k \rho)^{1 / 2} \cos \left(\frac{1}{2} \phi+\frac{1}{2} \phi^{\prime}\right)\right]\right\}+S(i k \rho),
$$

where for large $k \rho$

$$
S(i k \rho) \sim-\exp (-i k \rho-i \pi / 4) \sum_{n=0}^{\infty} B_{n} i^{n}(k \rho)^{-n-1 / 2}
$$

and these expressions are valid over the whole region of the wedge. The plus or minus sign has to be taken according as the acoustic or the electromagnetic case is considered. Obviously $S$ represents the deviation of the wedge field from the half-plane field and the expansions (69) and (72) represent asymptotic expansions for this deviation term. If $(\gamma \rho)^{1 / 2} \cos \left(\frac{1}{2} \phi \pm \frac{1}{2} \phi^{\prime}\right)$ and $(k \rho)^{1 / 2} \cos \left(\frac{1}{2} \varphi \pm \frac{1}{2} \phi^{\prime}\right)$ are large, i. e., for the far field at large distances from the boundaries of geometric optics one can replace the error function by its asymptotic expansion [9, p. 147],

$$
\operatorname{Erfc}(z) \sim \pi^{-1} \exp \left(-z^{2}\right) \sum_{n=0}^{\infty}(-1)^{n} \Gamma\left(n+\frac{1}{2}\right) z^{-2 n-1}, \quad-3 \pi / 4<\arg z<3 \pi / 4,
$$

and the asymptotic expansion of $U$ is of the same type as the one for $S$. On the boundary of the geometric reflection $\left(\phi+\phi^{\prime}=\pi\right),(71)$ becomes 


$$
U=\exp \left[i k \rho \cos \left(\phi-\phi^{\prime}\right)\right]\left\{1-\frac{1}{2} \operatorname{Erfc}\left[(2 i k \rho)^{1 / 2} \cos \left(\frac{1}{2} \phi-\frac{1}{2} \phi^{\prime}\right)\right]\right\} \mp \frac{1}{2} \exp (-i k \rho)+S(i k \rho) .
$$

On the boundary of the geometric shadow $\left(\phi-\phi^{\prime}=\pi\right)$,

$$
U=\frac{1}{2} \exp (-i k \rho) \mp \exp \left[\left(i k \rho \cos \left(\phi+\phi^{\prime}\right)\right] \cdot\left\{1-\frac{1}{2} \operatorname{Erfc}\left[(2 i k \rho)^{1 / 2} \cos \left(\frac{1}{2} \phi+\frac{1}{2} \phi^{\prime}\right)\right]\right\}+S(i k \rho) .\right.
$$

Using (73) these results can be written as

$$
\begin{aligned}
& U=\exp \left[i k \rho \cos \left(\phi-\phi^{\prime}\right)\right] \mp \frac{1}{2} \exp (-i k \rho)+O\left[(k \rho)^{-1 / 2}\right], \\
& U=\frac{1}{2} \exp (-i k \rho)+O\left[(k \rho)^{-\frac{1}{2}}\right] .
\end{aligned}
$$

These equations express the fact that the far field on the boundaries of geometric optics is independent of the angle of the wedge.

\section{Energy Radiated From a Hertz Dipole and From an Acoustic Point Source in the Presence of a Perfectly Reflecting Wedge or Corner}

Let the source $Q\left(\rho^{\prime}, \phi^{\prime}, 0\right)$ be a Hertz dipole with its axis parallel to the edge. The functions $f(\theta)$ and $H(x)$ are then

$$
\begin{gathered}
f(\theta)=\left(r^{2}+z^{2}\right)^{-1 / 2} \exp \left[-i k\left(r^{2}+z^{2}\right)^{1 / 2}\right], \\
r=\left(\rho^{2}+\rho^{\prime 2}-2 \rho \rho^{\prime} \cos \theta\right)^{1 / 2}, \\
H(x)=\left(\rho^{2}+\rho^{\prime 2}+2 \rho \rho^{\prime} \cosh x+z^{2}\right)^{-1 / 2} \exp \left[-i k\left(\rho^{2}+\rho^{\prime 2}+2 \rho \rho^{\prime} \cosh x+z^{2}\right)^{1 / 2}\right] .
\end{gathered}
$$

The function $U$ given by (28) (taken with the minus sign) represents the vector potential $A$ of the electromagnetic field. Its components are

$$
A_{x}=A_{y}=0, \quad A_{z}=U \text {. }
$$

With this relation the components $E_{x}, E_{y}, E_{z}$ of the electric field vector $E$ are known and the radiated energy can be determined by Poyntings method. The application of this method is very difficult in view of the complexity of the expression for $U$. It is therefore advisable to use the so called E. M. F. method for the determination of the radiation resistance $R$ of the dipole [37, p. 457] and [33, p. 134]. $R$ is given by

$$
R=-(L / J) \operatorname{Re}\left(E_{z}\right)_{D}
$$

Here $L$ and $J$ are the length and the current of the dipole respectively. $\operatorname{Re}\left(E_{z}\right)_{D}$ is the real part of the $z$ component of the electric field at the location of the dipole $Q\left(\rho^{\prime}, \phi^{\prime}, 0\right)$. It follows from (78) for the field,

$$
E_{z}=-i C\left(\frac{\partial^{2} U}{\partial z^{2}}+k^{2} U\right)
$$

$C$ is a constant (real $>0$ ) and is of no importance here since the "reduced" radiation resistance $R / R_{0}$ will be determined. This is the quotient of the radiation resistance $R$ for the complete system (dipole plus reflector) and the radiation resistance $R_{0}$ of the dipole in free space (without the presence of the reflector). At first the contribution of $F_{1}$ (which represents a set of dipoles consisting of the original source and a number of images) to $E_{z}$ is considered. From (30) and (76)

$$
\begin{aligned}
U_{1} & =F_{1}\left(\phi-\phi^{\prime}\right)-F_{1}\left(\phi+\phi^{\prime}\right) \\
& =\sum_{p=p_{1}}^{p_{2}}\left(r_{p}^{2}+z^{2}\right)^{-1 / 2} \exp \left[\left(-i k\left(r_{p}^{2}+z^{2}\right)^{1 / 2}\right]-\sum_{q=q_{1}}^{q_{2}}\left(r_{q}^{2}+z^{2}\right)^{-1 / 2} \exp \left[-i k\left(r_{q}^{2}+z^{2}\right)^{1 / 2}\right],\right. \\
r_{p}^{2} & =\rho^{2}+\rho^{\prime 2}-2 \rho \rho^{\prime} \cos \left(\phi-\phi^{\prime}+2 \alpha p\right)
\end{aligned}
$$




$$
\begin{aligned}
& r_{q}^{2}=\rho^{2}+\rho^{\prime 2}-2 \rho \rho^{\prime} \cos \left(\phi+\phi^{\prime}+2 \alpha q\right) \\
& p_{1}=-\left[\frac{\pi+\phi-\phi^{\prime}}{2 \alpha}\right], \quad p_{2}=\left[\frac{\pi-\phi+\phi^{\prime}}{2 \alpha}\right] \\
& q_{1}=-\left[\frac{\pi+\phi+\phi^{\prime}}{2 \alpha}\right], \quad q_{2}=\left[\frac{\pi-\phi-\phi^{\prime}}{2 \alpha}\right] .
\end{aligned}
$$

It is then easily found that

with

$$
\operatorname{Re}\left[-i C\left(\frac{\partial^{2} U_{1}}{\partial z^{2}}+k^{2} U_{1}\right)_{D}\right]=-C k^{3}\left[\sum_{n=n_{1}}^{n_{2}} g\left(k d_{n}\right)-\sum_{m=m_{1}}^{m_{2}} g\left(k d_{m}\right)\right]
$$

$$
\begin{gathered}
d_{n}=2 \rho^{\prime} \sin (\alpha n), \quad d_{m}=2 \rho^{\prime} \sin \left(\phi^{\prime}+\alpha m\right) \\
n_{1}=-\left[\frac{1}{2} \pi / \alpha\right], \quad n_{2}=\left[\frac{1}{2} \pi / \alpha\right], \\
m_{1}=-\left[\frac{\pi+2 \phi^{\prime}}{2 \alpha}\right], \quad m_{2}=\left[\frac{\pi-2 \phi^{\prime}}{2 \alpha}\right], \\
g(\zeta)=\zeta^{-1} \sin \zeta+\zeta^{-2} \cos \zeta-\zeta^{-3} \sin \zeta, \quad g(0)=2 / 3 .
\end{gathered}
$$

The term $n=0$ in the sum (83) represents the contribution of the original dipole source. It follows then for the radiation resistance of the Hertz dipole in free space by (83) and (79)

The contribution of $F_{2}$ to $U$ is

$$
R_{0}=2 L /(3 J) C k^{3} .
$$

and it follows by (31) and (77)

$$
U_{2}=F_{2}\left(\phi-\phi^{\prime}\right)-F_{2}\left(\phi+\phi^{\prime}\right)
$$

$$
\begin{aligned}
& \operatorname{Re}\left[-i C\left(\frac{\partial^{2} U_{2}}{\partial z^{2}}+k^{2} U_{2}\right)_{D}\right]=(2 \alpha)^{-1} C k^{3}\left\{2 \sin \left(\pi^{2} / \alpha\right) \int_{0}^{\infty} \frac{g\left(2 k \rho^{\prime} \cosh x / 2\right)}{\cosh (\pi x / \alpha)-\cos \left(\pi^{2} / \alpha\right)} d x\right. \\
& \left.\left.-\int_{0}^{\infty} g\left(2 k \rho^{\prime} \cosh \frac{x}{2}\right)\left[\frac{\sin \left[\frac{\pi}{\alpha}\left(\pi-2 \phi^{\prime}\right)\right]}{\cosh (\pi x / \alpha)-\cos \left[\frac{\pi}{\alpha}\left(\pi-2 \phi^{\prime}\right)\right]}+\frac{\sin \left[\frac{\pi}{\alpha}\left(\pi+2 \phi^{\prime}\right)\right]}{\cosh (\pi x / \alpha)-\cos \left[\frac{\pi}{\alpha}\left(\pi+2 \phi^{\prime}\right)\right.}\right]\right] d x\right\} .
\end{aligned}
$$

It follows, then, for the quotient of the radiation resistance $R$ of the dipole in the presence of the reflector and the radiation resistance $R_{0}$ of the dipole in free space by eq $(79),(83),(87)$, and $(85)$.

$$
\begin{aligned}
& R / R_{0}=3 / 2\left[\sum_{n=n_{1}}^{n_{2}} g\left(k d_{n}\right)-\sum_{m=m_{1}}^{m_{2}} g\left(k d_{m}\right)\right]-\frac{3}{4 \alpha}\left\{2 \sin \left(\pi^{2} / \alpha\right) \int_{0}^{\infty} \frac{g\left(2 k \rho^{\prime} \cosh \frac{x}{2}\right)}{\cosh (\pi x / \alpha)-\cos \left(\pi^{2} / \alpha\right)} d x\right. \\
& \left.-\int_{0}^{\infty} g\left(2 k \rho^{\prime} \cosh \frac{x}{2}\right)\left[\frac{\sin \left[\frac{\pi}{\alpha}\left(\pi-2 \phi^{\prime}\right)\right]}{\cosh (\pi x / \alpha)-\cos \left[\frac{\pi}{\alpha}\left(\pi-2 \phi^{\prime}\right)\right]}+\frac{\sin \left[\frac{\pi}{\alpha}\left(\pi+2 \phi^{\prime}\right)\right]}{\cosh (\pi x / \alpha)-\cos \left[\frac{\pi}{\alpha}\left(\pi+2 \phi^{\prime}\right)\right]}\right] d x\right\} .
\end{aligned}
$$

The occurring parameters and $g(\zeta)$ are given in (84). Since $g(\zeta)$ vanishes for large $\zeta$ it is clear that if the distance of the dipole from the walls is large $\left(\rho^{\prime} \rightarrow \infty\right)$ only the term $n=0$ in the sum gives a contribution, and then

$$
\lim R / R_{0}=3 / 2 g(0)=1 \text {. }
$$


The formula (88) is valid for an arbitrary $\alpha$. But if $\alpha>\pi$, i. e., in the case of a wedge reflector (figs. 2 and 3 ), the two sums on the r. h. s. of (88) can be given explicitly. By (84) the limits $n_{1}, n_{2}, m_{1}, m_{2}$, of the sums in case $\alpha>\pi$ are

$$
\begin{array}{ll}
n_{1}=n_{2}=m_{1}=m_{2}=0 & \text { for } 0<\phi^{\prime}<\frac{1}{2} \pi, \\
n_{1}=n_{2}=m_{1}=0, \quad m_{2}=-1 & \text { for } \frac{1}{2} \pi<\phi^{\prime}<\frac{1}{2} \alpha .
\end{array}
$$

Thus

$$
\begin{aligned}
\sum_{n=n_{1}}^{n_{2}} g\left(k d_{n}\right)-\sum_{m=m_{1}}^{m_{2}} g\left(k d_{m}\right) & =2 / 3-g\left(2 k \rho^{\prime} \sin \phi^{\prime}\right), & & \text { if } 0<\phi^{\prime}<\frac{1}{2} \pi, \\
& =2 / 3, & & \text { if } \frac{1}{2} \pi<\phi^{\prime}<\frac{1}{2} \alpha .
\end{aligned}
$$

The analysis is exactly the same when the source $Q\left(\rho^{\prime}, \phi, 0\right)$ is an acoustic point source. In this case the plus sign in $(28)$ has to be taken. The function $g(\zeta)$ in $(84)$ which characterizes the coupling term between two Hertz dipoles (parallel to each other and the line connecting their centers perpendicular to their axis) has to be replaced by the corresponding coupling term $g_{1}(\zeta)$ for two acoustic point sources [30].

$$
g_{1}(\zeta)=\zeta^{-1} \sin \zeta
$$

In this case the corresponding formula for the relative radiation resistance as defined before is

$$
\begin{aligned}
R / R_{0} & =\sum_{n=n_{1}}^{n_{2}} g_{1}\left(k d_{n}\right)+\sum_{m=m^{\prime}}^{m_{2}} g_{1}\left(k d_{m}\right)-(2 \alpha)^{-1}\left\{2 \sin \left(\pi^{2} / \alpha\right) \int_{0}^{\infty} \frac{g_{1}\left(2 k \rho^{\prime} \cosh \frac{x}{2}\right)}{\cosh (\pi x / \alpha)-\cos \left(\pi^{2} \alpha\right)} d x\right. \\
& \left.+\int_{0}^{\infty} g_{1}\left(2 k \rho^{\prime} \cosh \frac{x}{2}\right)\left[\frac{\sin \left[\frac{\pi}{\alpha}\left(\pi-2 \phi^{\prime}\right)\right]}{\cosh (\pi x / \alpha)-\cos \left[\frac{\pi}{\alpha}\left(\pi-2 \rho^{\prime}\right)\right]}+\frac{\sin \left[\frac{\pi}{\alpha}\left(\pi+2 \phi^{\prime}\right)\right]}{\cosh (\pi x / \alpha)-\cos \left[\frac{\pi}{\alpha}\left(\pi+2 \phi^{\prime}\right)\right]}\right] d x\right\} .
\end{aligned}
$$

The definition of the $n_{1}, n_{2}, m_{1}, m_{2}, d_{n}, d_{m}$ is the same as in (84), while $g_{1}(\zeta)$ is given by (89). If $\alpha>\pi$, then as before

$$
\begin{array}{ll}
n_{1}=n_{2}=m_{1}=m_{2}=0 & \text { for } 0<\phi^{\prime}<\frac{1}{2} \pi, \\
n_{1}=n_{2}=m_{1}=0, \quad m_{2}=-1 & \text { for } \frac{1}{2} \pi<\phi^{\prime}<\frac{1}{2} \alpha .
\end{array}
$$

and

$$
\begin{aligned}
\sum_{n=n_{1}}^{n_{2}} g_{1}\left(k d_{n}\right)+\sum_{m=m_{1}}^{m_{2}} g_{1}\left(k d_{m}\right) & =1+g_{1}\left(2 k \rho^{\prime} \sin \phi^{\prime}\right), & & 0<\phi^{\prime}<\frac{1}{2} \pi, \\
& =1, & & \frac{1}{2} \pi^{\prime}<\phi^{\prime}<\frac{1}{2} \alpha .
\end{aligned}
$$

\section{Transient Solutions}

The results obtained in section 2 shall now be used to investigate the corresponding pulse problems. The majority of the contributions quoted at the beginning of this paper are based upon a direct attack on the time dependent wave equation. Integral transform methods were used in [38] (Kontorovich-Lebedev transform) and [40] (Fourier transform). In the cases considered here the application of Laplace's transform leads rapidly to the desired results. Let the excitation of the source be represented by a pulse starting at the time $t=0$. Let the pulse function be $g(t)$, and $g(t)=0$ for $t<0$. Let the pulse function be such that it admits its representation by Laplace's integral formula: 


$$
g(t)=(2 \pi i)^{-1} \int_{c-i \infty}^{c+i \infty}\left[\int_{0}^{\infty} g(\tau) \exp \left(-\gamma_{\tau}\right) d \tau\right] \exp (\gamma t) d \gamma
$$

In section 2, various expressions $U$ for the exponential decay problem were given. This case corresponded according to (5) to the time dependency $\exp (\gamma t)$, (put $c=1$ ). It can then be concluded that for a pulse excitation $g(t)$ as represented by (91) the expression for the field is represented by

$$
\Phi(t)=(2 \pi i)^{-1} \int_{c-i \infty}^{c+i \infty}\left[\int_{0}^{\infty} g(\tau) \exp (-\gamma \tau) d \tau\right] U \exp (\gamma t) d \gamma .
$$

The solution of the pulse problem is therefore given as the inverse Laplace transformation of

$$
U \int_{0}^{\infty} g(\tau) \exp (-\gamma \tau) d \tau
$$

with respect to $\gamma$.

\subsection{Special Cases for $g(t)$}

For a unit step function $g(t)=1$, the field (92) becomes

$$
\Phi(t)=(2 \pi i)^{-1} \int_{c-i \infty}^{c+i \infty} U \gamma^{-1} \exp (\gamma t) d \gamma
$$

For a "Dirac" pulse excitation imposed at the time $t=t_{1}>0$ : $g(t)=\delta\left(t-t_{1}\right)$, one finds first

$$
\int_{0}^{\infty} g(\tau) \exp (-\gamma \tau) d \tau=\int_{0}^{\infty} \delta\left(\tau-t_{1}\right) \exp (-\gamma \tau) d \tau=\exp \left(-\gamma t_{1}\right) .
$$

The field due to a "Dirac" pulse excitation becomes then

$$
\Phi_{D}\left(t-t_{1}\right)=(2 \pi i)^{-1} \int_{c-i \infty}^{c+i \infty} U \exp \left[\gamma\left(t-t_{1}\right)\right] d \gamma .
$$

If the order of integration in (92) can be interchanged, it results in

$$
\Phi(t)=\int_{0}^{\infty} g(\tau)\left\{(2 \pi i)^{-1} \int_{c-i \infty}^{c+i \infty} U \exp [\gamma(t-\tau)] d \gamma\right\} d \tau
$$

Since by (96) the inner integral is the effect of a "Dirac" pulse, excitation (97) can be written as

$$
\Phi(t)=\int_{0}^{\infty} g(\tau) \Phi_{D}(t-\tau) d \tau
$$

i. e., the field due to an excitation $g(t)$ of the source $Q$ can be obtained by an integration process involving the field due to a "Dirac" pulse excitation. The general equations (96), (98) shall now be applied to the problem of the diffraction of arbitrary pulses (plane, cylindrical, and spherical) incident on a perfectly reflecting wedge or corner. The case of "Dirac" pulse excitation imposed at the time $t=0$ shall be considered first. The Laplace inversion of a function $h(\gamma)$ with respect to $\gamma$ shall be denoted by $\bar{h}(t)$, i. e.,

$$
\bar{h}(t)=(2 \pi i)^{-1} \int_{c-i \infty}^{c+i \infty} h \exp (\gamma t) d \gamma .
$$

It follows from (96) and (28) for the field $\Phi_{D}$ due to a plane, cylindrical or spherical "Dirac" pulse: 


$$
\begin{aligned}
\Phi_{D}(t) & =\bar{F}\left(\phi-\phi^{\prime}\right) \mp \bar{F}\left(\phi+\phi^{\prime}\right) \\
& =\sum_{n=n_{1}}^{n_{2}} \bar{f}\left(\phi-\phi^{\prime}+2 \alpha n\right) \mp \sum_{m=m_{1}}^{m_{2}} \bar{f}\left(\phi+\phi^{\prime}+2 \alpha m\right)+\int_{0}^{\infty} \bar{H}(x)\left[G\left(\phi-\phi^{\prime}\right) \mp G\left(\phi+\phi^{\prime}\right)\right] d x,
\end{aligned}
$$

where

$$
\begin{gathered}
n_{1}=-\left[\frac{\pi+\phi-\phi^{\prime}}{2 \alpha}\right], \quad n_{2}=\left[\frac{\pi-\phi-\phi^{\prime}}{2 \alpha}\right], \quad m_{1}=-\left[\frac{\pi+\phi+\phi^{\prime}}{2 \alpha}\right], \quad m_{2}=\left[\frac{\pi-\phi-\phi^{\prime}}{2 \alpha}\right], \\
G(\theta) \equiv-(2 \alpha)^{-1}\left\{\frac{\sin \left[\frac{\pi}{\alpha}(\pi-\theta)\right]}{\cosh (\pi x / \alpha)-\cos \left[\frac{\pi}{\alpha}(\pi-\theta)\right]^{+}} \frac{\sin \left[\frac{\pi}{\alpha}(\pi+\theta)\right]}{\cosh (\pi x / \alpha)-\cos \left[\frac{\pi}{\alpha}(\pi+\theta)\right]}\right] .
\end{gathered}
$$

The respective Laplace inversions $\bar{f}(\theta)$ and $\bar{H}(x)$ are for a plane pulse:

$$
\begin{aligned}
\bar{f}(\theta) & =\delta(t+\rho \cos \theta) \\
\bar{H}(x) & =\delta(t-\rho \cosh x) .
\end{aligned}
$$

For a cylindrical pulse one has

$$
\begin{aligned}
\bar{f}(\theta) & =2 i \pi^{-1}\left(t^{2}-\rho^{2}-\rho^{\prime 2}+2 \rho \rho^{\prime} \cos \theta\right)^{-1 / 2}, & & \text { for } t>\left(\rho^{2}+\rho^{\prime 2}-2 \rho \rho^{\prime} \cos \theta\right)^{1 / 2}, \\
& =0, & & \text { otherwise. } \\
\bar{H}(x) & =2 i \pi^{-1}\left(t^{2}-\rho^{2}-\rho^{\prime 2}-2 \rho \rho^{\prime} \cosh x\right)^{-1 / 2}, & & \text { for } 2 \rho \rho^{\prime} \cosh x<t^{2}-\rho^{2}-\rho^{\prime 2}, \\
& =0, & & \text { otherwise. }
\end{aligned}
$$

Finally, for a spherical pulse

$$
\begin{gathered}
\bar{f}(\theta)=\left(r^{2}+z^{2}\right)^{-1 / 2} \delta\left[t-\left(r^{2}+z^{2}\right)^{1 / 2}\right] \\
\bar{H}(x)=\left(\rho^{2}+\rho^{\prime 2}+z^{2}+2 \rho \rho^{\prime} \cosh x\right)^{-1 / 2} \delta\left[t-\left(\rho^{2}+\rho^{\prime 2}+z^{2}+2 \rho \rho^{\prime} \cosh x\right)^{1 / 2}\right] .
\end{gathered}
$$

The Laplace inversions for $f(\theta)$ and $H(x)$ are obtained from (95) for the plane and for the spherical pulse, while [10, vol. 1, p. 277, formula 8] gives the inversion for the cylindrical pulse. It does not seem possible to express the integral $\int_{0}^{\infty} \bar{H}(x) G(\theta) d x$ in a closed form in the case of a cylindrical pulse. To obtain the field due to a pulse excitation $g(t)$, the equations (98) and (100) have to be used. For a plane and a spherical pulse the integration process in (98) can be performed immediately. The results are

$$
\begin{aligned}
\Phi(t)=\sum_{n=n_{1}}^{n_{2}} g\left[t+\rho \cos \left(\phi-\phi^{\prime}+2 \alpha n\right)\right] \mp & \sum_{m=m_{1}}^{m_{2}} g\left[t+\rho \cos \left(\phi+\phi^{\prime}+2 \alpha m\right)\right] \\
& +\int_{0}^{\cosh x=t / \rho} g(t-\rho \cosh x)\left[G\left(\phi-\phi^{\prime}\right) \mp G\left(\phi+\phi^{\prime}\right)\right] d x,
\end{aligned}
$$

for the plane pulse.

$$
\begin{aligned}
\Phi(t) & =\sum_{n=n_{1}}^{n_{2}} R_{n}^{-1} g\left(t-R_{n}\right) \mp \sum_{m=m_{1}}^{m_{2}} R_{m}^{-1} g\left(t-R_{m}\right) \\
& +\int_{0}^{x_{1}}\left(\rho^{2}+\rho^{\prime 2}+z^{2}+2 \rho \rho^{\prime} \cosh x\right)^{-1 / 2} g\left[t-\left(\rho^{2}+\rho^{\prime 2}+z^{2}+2 \rho \rho^{\prime} \cosh x\right)^{1 / 2}\right]\left\{G\left(\phi-\phi^{\prime}\right) \mp G\left(\phi+\phi^{\prime}\right)\right\} d x,
\end{aligned}
$$

for the spherical pulse.

The upper limit $x_{1}$ of the integral is given by

$$
2 \rho \rho^{\prime} \cosh x_{1}=t^{2}-\rho^{2}-\rho^{\prime 2}-z^{2} \text { for (109), and } \cosh x_{1}=t / \rho \text { for }(108) \text {. }
$$




$$
\begin{aligned}
& R_{n}=\left[\rho^{2}+\rho^{\prime 2}+z^{2}-2 \rho \rho^{\prime} \cos \left(\phi-\phi^{\prime}+2 \alpha n\right)\right]^{1 / 2} \\
& R_{m}=\left[\rho^{2}+\rho^{\prime 2}+z^{2}-2 \rho \rho^{\prime} \cos \left(\phi+\phi^{\prime}+2 \alpha m\right)\right]^{1 / 2} .
\end{aligned}
$$

The other properties are given in (101). Again in the case of a wedge $(\alpha>\pi)$, the properties $n_{1}, n_{2}, m_{1}, m_{2}$ are given in section 3 . The representation of the cylindrical pulse field leads to a double integral. For the special case of a half plane, however, the integral occurring in (100) can be solved. In the following some special cases are considered in which the solution for the transient field become elementary.

\subsection{Special Cases}

\section{a. Cylindrical Pulse and Half Plane}

The case of a line source $Q$ parallel to the edge of a perfectly reflecting half plane is considered. The regions I, II, III as described in section 3 are indicated in figure 6 . For a half plane $(\alpha=2 \pi)$, one obtains from (101),

$$
G(\theta)=-\pi^{-1} \cos \left(\frac{1}{2} \theta\right) \cosh \left(\frac{1}{2} x\right)(\cosh x+\cos \theta)^{-1} .
$$

It follows then for a cylindrical "Dirac" pulse by (105),

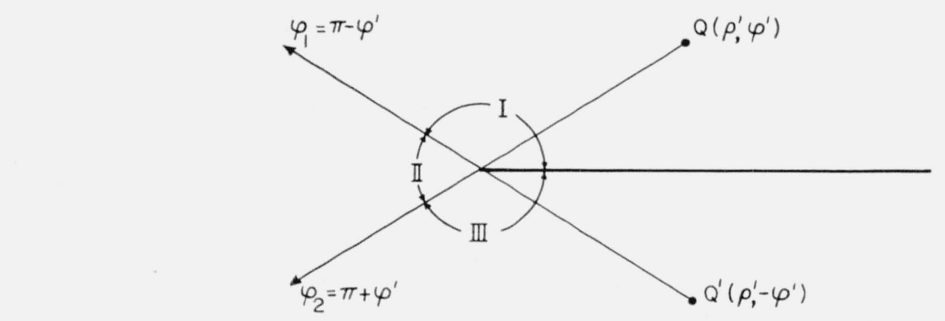

FIGURE 6.

$$
\int_{0}^{\infty} \bar{H}(x) G(\theta) d x=-2 i \pi^{-2} \cos \left(\frac{1}{2} \theta\right) \int_{0}^{x_{1}} \cosh \left(\frac{1}{2} x\right) \frac{\left(t^{2}-\rho^{2}-\rho^{\prime 2}-\rho \rho^{\prime} \cosh x\right)^{-1 / 2}}{\cosh x+\cos \theta} d x .
$$

The upper limit $x_{1}$ of the integral is given by $2 \rho \rho^{\prime} \cosh x_{1}=t^{2}-\rho^{2}-\rho^{\prime 2}$, and from (105) it is obvious that the integral is only different from zero when $t>\rho+\rho^{\prime}$. The substitution cosh $x=1+v^{2}, \cosh \left(\frac{1}{2} x\right) d x=2^{1 / 2} d v$ gives for the integral

$$
\begin{aligned}
\int_{0}^{\infty} \bar{H}(x) G(\theta) d x & =-i \pi^{-1} \cos \left(\frac{1}{2} \theta\right)\left|\sec \left(\frac{1}{2} \theta\right)\right|\left(t^{2}-\rho^{2}-\rho^{\prime 2}+2 \rho \rho^{\prime} \cos \theta\right)^{-1 / 2}, & \text { if } t>\rho+\rho^{\prime}, \\
& =0, & \text { otherwise. }
\end{aligned}
$$

It follows then from (100) by means of (35), (36), (37), (104), and (113) for the field due to a cylindrical "Dirac" pulse incident on a half plane

$$
\begin{aligned}
\Phi_{D}(t) & =2 i \pi^{-1}\left\{\left(t^{2}-R_{1}^{2}\right)^{-1 / 2} \mp\left(t^{2}-\mathrm{R}_{2}^{2}\right)^{-1 / 2}-\frac{1}{2}\left[\left(t^{2}-R_{1}^{2}\right)^{-1 / 2} \mp\left(t^{2}-R_{2}^{2}\right)^{-1 / 2}\right]\right\} & & \text { for region } \mathrm{I}, \\
& =2 i \pi^{-1}\left\{\left(t^{2}-\mathrm{R}_{1}^{2}\right)^{-1 / 2}-\frac{1}{2}\left[\left(t^{2}-\mathrm{R}_{1}^{2}\right)^{-1 / 2} \pm\left(t^{2}-R_{2}^{2}\right)^{-1 / 2}\right]\right\}, & & \text { for region II, } \\
& =i \pi^{-1}\left[\left(t^{2}-R_{1}^{2}\right)^{-1 / 2} \mp\left(t^{2}-R_{2}^{2}\right)^{-1 / 2}\right], & & \text { for region III, }
\end{aligned}
$$

where

$$
R_{1}^{2}=\rho^{2}+\rho^{\prime 2}-2 \rho \rho^{\prime} \cos \left(\phi-\phi^{\prime}\right), \quad R_{2}^{2}=\rho^{2}+\rho^{\prime 2}-2 \rho \rho^{\prime} \cos \left(\phi+\phi^{\prime}\right) .
$$

In (114), $\left(t^{2}-R_{1_{0} 2}^{2}\right)^{-1 / 2}=0$ for $t<\rho+\rho^{\prime}$ when this expression is within the brackets; otherwise zero for $t<R_{1,2}$. The expression (114) is elementary in all the three regions. Former investigations [38] established $\Phi_{D}(t)$ as an infinite series involving Legendre functions. (It 
can be shown that this series can be summed and leads to the expressions in (114).) (114) and (98) it follows for the field $\Phi$ due to an excitation $g(t)$,

$$
\begin{aligned}
& \Phi(t)=2 i \pi^{-1}\left\{\int_{0}^{t-R_{1}}\left[(t-\tau)^{2}-R_{1}^{2}\right]^{-1 / 2} g(\tau) d \tau \mp \int_{0}^{t-R_{2}}\left[(t-\tau)^{2}-R_{2}^{2}\right]^{-1 / 2} g(\tau) d \tau\right. \\
& \left.-\frac{1}{2} \int_{0}^{t-\rho-\rho^{\prime}}\left[\left((t-\tau)^{2}-R_{1}^{2}\right)^{-1 / 2} \mp\left((t-\tau)^{2}-R_{2}^{2}\right)^{-1 / 2}\right] g(\tau) d \tau\right\}, \quad \text { region } \mathrm{I} \text {. } \\
& \Phi(t)=2 i \pi^{-1}\left\{\int_{0}^{t-R_{1}}\left[(t-\tau)^{2}-R_{1}^{2}\right]^{-1 / 2} g(\tau) d \tau\right. \\
& \left.-\frac{1}{2} \int_{0}^{t-\rho-\rho^{\prime}}\left[\left((t-\tau)^{2}-R_{1}^{2}\right)^{-1 / 2} \pm\left((t-\tau)^{2}-R_{2}^{2}\right)^{-1 / 2}\right] g(\tau) d \tau\right\}, \quad \text { region II. } \\
& \Phi) t)=i \pi^{-1} \int_{0}^{t-\rho-\rho^{\prime}}\left[\left((t-\tau)^{2}-R_{1}^{2}\right)^{-1 / 2} \mp\left((t-\tau)^{2}-R_{2}^{2}\right)^{-1 / 2}\right] g(\tau) d \tau, \quad \text { region III. }
\end{aligned}
$$

\section{b. Plane Unit Step Function Pulse and Wedge or Corner}

Now, $g(t)=1$ for $t>0$, and $g(t)=0$ for $t<0$. Then the terms of the sums on the r. h. s. of (108) are either zero or one according as the argument of $g$ is negative or positive. The well-known formula

$$
\int[\cosh (b x)-a]^{-1} d x=2 b^{-1}\left(1-a^{2}\right)^{-1 / 2} \arctan \left[\left(\frac{1+a}{1-a}\right)^{1 / 2} \tanh \left(\frac{1}{2} b x\right)\right]
$$

gives for the integral in (108) by (101)

$$
\begin{aligned}
& \int_{0}^{\cosh x=t / \rho} G(\theta) d x=-\pi^{-1} \arctan \left\{\frac{\left[t+\left(t^{2}-\rho^{2}\right)^{1 / 2}\right]^{\pi / \alpha}-\rho^{\pi / \alpha}}{\left[t+\left(t^{2}-\rho^{2}\right)^{1 / 2}\right]^{\pi / \alpha}+\rho^{\pi / \alpha}} \operatorname{cotan}\left[\frac{\pi}{2 \alpha}(\pi-\theta)\right]\right\} \\
&-\pi^{-1} \arctan \left\{\frac{\left[t+\left(t^{2}-\rho^{2}\right)^{1 / 2}\right]^{\pi / \alpha}-\rho^{\pi / \alpha}}{\left[t+\left(t^{2}-\rho^{2}\right)^{1 / 2}\right]^{\pi / \alpha}+\rho^{\pi / \alpha}} \operatorname{cotan}\left[\frac{\pi}{2 \alpha}(\pi+\theta)\right]\right\}
\end{aligned}
$$

for $t>\rho$ and equal to zero for $t<\rho$. Thus the field (108) for a unit step function excitation $[19,24]$ is elementary.

\section{c. Spherical Unit Step Function Pulse and Half Plane}

The center of the spherical pulse is $Q$ (fig. 6). Again, $g(t)=1$. The excited field is given by (109) and $G(\theta)$ is given by (112). The integral occuring in 109) is of the form

$$
\begin{aligned}
\int_{0}^{x_{1}}\left(\rho^{2}+\rho^{\prime 2}\right. & +2 \rho \rho \cosh x)^{-1 / 2} G(\theta) d x \\
& =-\pi^{-1} \cos \left(\frac{1}{2} \theta\right) \int_{0}^{x_{1}} \cosh \left(\frac{1}{2} x\right)(\cosh x+\cos \theta)^{-1}\left(\rho^{2}+\rho^{\prime 2}+z^{2}+2 \rho \rho^{\prime} \cosh x\right)^{-1 / 2} d x \\
& =-2^{1 / 2} \pi^{-1} \cos \left(\frac{1}{2} \theta\right) \int_{0}^{v_{1}}\left(1+\cos \theta+v^{2}\right)^{-1}\left[\left(\rho+\rho^{\prime}\right)^{2}+z^{2}+2 \rho \rho^{\prime} v^{2}\right]^{-1 / 2} d v
\end{aligned}
$$

using the substitution $\cosh x=1+v^{2}, \quad \cosh \left(\frac{1}{2} x\right) d x=2^{1 / 2} d v$. The upper limit $v_{1}$ is given by $v_{1}=\left[t^{2}-\left(\rho+\rho^{\prime}\right)^{2}-z^{2}\right]^{\frac{1}{2}}$. Again this integral is elementary. Each sum in (109) reduces to at most one elementary term (see the tables in section 3), and the resulting field [40] is elementary.

It should be remembered that the previous investigations were based on the assumption that the velocity of the field is unity. The time parameter $t$ has therefore to be replaced by $c t$ when the velocity of the field is equal to $c$. The expression $U$ for the time harmonic field as given in section 2 can easily be regained as the limiting case of a transient field $\Phi$ due to an excitation $g(t)=\exp (i \omega t)$. 


\section{'7. References}

[1] T. J. Bromwich, Diffraction of waves by a wedge, Proc. London Math. Soc. 14, 450-463 (1915).

[2] T. J. Bromwich, An introduction to the theory of infinite series (MacMillan \& Co., Ltd., London, 1931)

[3] H. S. Carslaw, Some multiform solutions of the partial differential equations of physies and mathematics and their applications, Proc. London Math. Soc. 30, 121-163 (1899).

[4] H. S. Carslaw, Diffraction of waves by a wedge of any angle, Proc. London Math. Soc. 18, 291-306 (1920).

[5] P. C. Clemmow, A note on the diffraction of a cylindrical wave by a perfectly conducting half plane, Quart. J. Mech. Appl. Math. 3, 377-384 (1950).

[6] P. C. Clemmow and C. M. Munford, A table of $\sqrt{\frac{1}{2}} \pi \exp \left(\frac{1}{2} i \pi \rho^{2}\right) \int_{\rho}^{\infty} \exp \left(-\frac{1}{2} i \pi \lambda^{2}\right) d \lambda$ for complex values of $\rho$. Philos. Trans. Roy. Soc. London [A] 245, 189-211 (1953).

[7] E. T. Copson, On an integral equation arising in the theory of diffraction, Quart. J. Math. Oxford Ser. 17, 19-34 (1946).

[8] G. Doetsch, Handbuch der Laplэce Transformation, Vol. 2 (Birkhauser Verlag, Basel, 1955).

[9] A. Erdelyi, Higher transcendentel functions, Vol. 2 (McGraw-Hill Book Co., Inc., New York, N. Y., 1953).

[10] A. Erdelyi, Tables of integral transforms, 2 vols. (McGraw-Hill Book Co., Inc., New York, N. Y., 1954).

[11] F. G. Friedlander, The diffraction of sound pulses, Proc. Roy. Soc. London, [A]186, 322-351 (1946).

[12] F. G. Friedlander, On the half plane diffraction problem, Quart. J. Mech. Appl. Math. 4, 344-357 (1951).

[13] R. C. Gast, Half plane diffraction with line source excitation, Tech. Report No. 24, Carnegie Institute of Technology (1956).

[14] R. F. Harrington, Current element near the edge of a conducting half plane, J. Appl. Phys. 24, 547-550 (1953).

[15] A. E. Heins, The excitation of a perfectly conducting half plane by a dipole field. Tech. Report No. 17, Carnegie Institute of Technology (1955).

[16] G. Herglotz, Die Greensche Funktion der Wellengleichung für eine keilformige Begrenzung, Math. Ann. 124, 219-234 (1952).

[17] S. Karp, Separation of variables and Wiener-Hopf techniques, New York Univ. Math. Research Group, Research Rept. No. EM 25 (1950).

[18] I. Kay, The diffraction of an arbitrary pulse by a wedge, Comm. Pure Appl. Math. 6, 419-434 (1953).

[19] J. B. Keller and A. Blank, Diffraction and reflection of pulses by wedges and corners, Comm. Pure Appl. Math. 4, 75-94 (1951).

[20] M. J. Kontorovich and N. N. Lebedev, On a method of solution of some problems in the diffraction theory, J. Phys. Moseow, 1, 229-241 (1939).

[21] H. Lamb, On the diffraction of a solitary wave, Proc. London Math. Soc. 8, 422-437 (1910).

[22] H. M. MacDonald, A class of diffraction problems, Proc. London Math. Soc. 14, 410-427 (1915).

[23] W. Magnus, Über (ie Beugung elektromagnetischer Wellen an einer Halbebene, A. Phys. 17g, 168-179 (1941)

[24] J. W. Miles, On the diffraction of an acoustic pulse by a wedge, Proc. Roy. Soc. London [A] 212, 543 (1952)

[25] J. W. Miles, On the diffraction of an electromagnetic pulse by a wedge, Proc. Roy. Soc. London [A] 212, $547-551(1952)$

[26] F. Oberhettinger, Diffraction of waves by a wedge, Comm. Pure Appl. Math. 7, 551-563 (1954).

[27] F. Oberhettinger, On asymptotic series for functions occurring in the theory of diffraction of waves by wedges, J. Math. Physics, 34, 245-255 (1956).

[28] F. Oberhettinger, On the propagation of pulses, Z. Physik 146, 423-435 (1956).

[29] W. Pauli, On asymptotic series for functions in the theory of diffraction cf light, Phys. Rev. 54, 924-931 (1938).

[30] Rayleigh, Lord, On the production and distribution of sound, Phil. Mag. 6, 289-305 (1903).

[31] F. Rellich, Über das asymptotische Verhalten der Lösungen von $\Delta u+\lambda u=0$ in unendlichen Gebieten, Jber. Deutsch. Math. Verein 53, 57-65 (1943).

[32] T. B. A, Senior, The diffraction of a dipole field by a perfectly conducting half plane, Quart. J. Mech. Appl. Math. 6, 101-114 (1953).

[33] S. A. Schelkunoff, Electromagnetic waves (D. Van Nostrand Co., Inc., New York, N. Y., 1943).

[34] A. Sommerfeld, Mathematische Theorie der Diffraktion, Math. Ann. 47, 317-341 (1896).

[35] A. Sommerfeld, Theoretisches uber die Beugung der Roentgenstrahlen, Z. Math. Phys. 46, 11-97 (1901).

[36] A. Sommerfeld, Asymptotische Darstellung von Formeln aus der Beugungstheorie des Lichtes, J. reine angew. Math. 158, 199-208 (1928).

[37] J. A. Stratton, Electromagnetic theory (MeGraw-Hill Book Co., Inc., New York, N. Y., 1941).

[38] R. D. Turner, The diffraction of a cylindrical pulse by a half plane, Quart. Appl. Math. 14, 63-73 (1956).

[39] Y. V. Vandakurov, Diffraction by a perfectly conducting half plane of electromagnetic waves emitted by an arbitrarily oriented electric and magnetic dipole, Zh. Eks. Teor. Fiz. 26, 3-18 (1954). 
[40] J. Wait, Diffraction of a spherical wave pulse by a half screen, Canadian J. Phys. 35, 693-696 (1957).

[41] J. Wait, On the theory of an antenna with infinite corner reflector, Canadian J. Phys. 32, 365-371 (1954).

[42] H. Weyl, Ausbreitung elektromagnetischer Wellen über einem ebenen Leiter, Ann. Phys. 60, 481-500 (1919).

[43] F. J. Whipple, Diffraction by a wedge and kindred problems, Proc. London Math. Soc. 16, 94-111 (1917).

[44] A. Wiegrefe, Über einige mehrwertige Lösungen der Wellengleichung $\Delta u+k^{2} u=0$ und ihre Anwendungen in der Beugungstheorie, Ann. Phys. 39, 449-484 (1912).

To prove

\section{Appendix}

$$
\begin{gathered}
\pi I_{\nu}(a) K_{\nu}(b)=\int_{0}^{\pi} K_{0}\left[\left(a^{2}+b^{2}-2 a b \cos t\right)^{1 / 2}\right] \cos (\nu t) d t-\sin (\pi \nu) \\
\cdot \int_{0}^{\infty} \exp (-\nu t) K_{0}\left[\left(a^{2}+b^{2}+2 a b \cosh t\right)^{1 / 2}\right] d t, \quad a<b, \\
K_{\nu}(a) K_{\nu}(b)=\int_{0}^{\infty} K_{0}\left[\left(a^{2}+b^{2}+2 a b \cosh t\right)^{1 / 2}\right] \cosh (\nu t) d t, \\
K_{0}\left[\left(a^{2}+b^{2}-2 a b \cos \phi\right)^{1 / 2}\right]=2 \pi^{-1} \int_{0}^{\infty} K_{i \nu}(a) K_{i \nu}(b) \cosh [\nu(\pi-|\phi|)] d \nu, \quad 0 \leqq \operatorname{Re} \phi \leqq 2 \pi,
\end{gathered}
$$

one uses the formulas

$$
\begin{gathered}
\pi I \nu(z)=\int_{0}^{\pi} \exp (z \cos t) \cos (\nu t) d t-\sin (\pi \nu) \int_{0}^{\infty} \exp (-z \cosh t-\nu t) d t \quad[9, \text { p. 21] } \\
K_{\nu}(z)=\frac{1}{2} \int_{0}^{\infty} x^{-\nu-1} \exp \left(-x-\frac{1}{4} z^{2} / x\right) d x, \quad[9, \text { p. 82] } \\
I_{\nu}(a) K_{\nu}(b)=\frac{1}{2} \int_{0}^{\infty} x^{-1} \exp \left(-x-\frac{a^{2}+b^{2}}{4 x}\right) I_{\nu}\left(\frac{1}{2} a b / x\right) d x, \quad[9, \text { p. 53]. }
\end{gathered}
$$

\subsection{Proof of $(\mathrm{Al})$}

Starting with (A6) and replacing the modified Bessel function in the integrand by (4), one obtains, interchanging the order of integration,

$$
\begin{aligned}
\pi I_{\nu}(a) K_{\nu}(b)=\frac{1}{2} \int_{0}^{\pi} \cos (\nu t)\left[\int_{0}^{\infty} x^{-1} \exp \left(-x-\frac{a^{2}+b^{2}-2 a b \cos t}{4 x}\right) d x\right] d t \\
\quad-\frac{1}{2} \sin (\pi \nu) \int_{0}^{\infty} \exp (-\nu t)\left[\int_{0}^{\infty} x^{-1} \exp \left(-x-\frac{a^{2}+b^{2}+2 a b \cosh t}{4 x}\right) d x\right] d t .
\end{aligned}
$$

The inner integrals on the r. h. s. are known by (A5). This proves (A1).

The relation

\subsection{Proof of (A2)}

$$
K_{\nu}(z)=\frac{1}{2} \pi \sec (\pi \nu)\left[I_{-\nu}(z)-I_{\nu}(z)\right]
$$

together with (A1) gives (A2).

\subsection{Proof of (A3)}

Replace in (A2) $\nu$ by iv and obtain by Fourier's inversion theorem

$$
K_{0}\left[\left(a^{2}+b^{2}+a b \cosh t\right)^{1 / 2}\right]=2 \pi^{-1} \int_{0}^{\infty} K_{i v}(a) K_{i v}(b) \cos (\nu t) d \nu
$$


Replace here $t$ by $i(\pi-\phi)$ and obtain (A3). The restriction $0 \leq \operatorname{Re} \phi \leq 2 \pi$ is necessary to ensure convergence of the integral in (A3) at its upper limit, since for fixed real $x$ and large positive $\nu \gg x[9$, p. 88 , formula 19$]$,

$$
K_{i \nu}(x)=(2 \pi)^{1 / 2}\left(\nu^{2}-x^{2}\right)^{-1 / 4} \exp \left(-\frac{1}{2} \pi \nu\right)\left\{\sin \left[\left[\nu \cosh ^{-1}(\nu / x)-\left(\nu^{2}-x^{2}\right)^{1 / 2}+\frac{\pi}{4}\right]+O\left(\nu^{-1}\right)\right\} .\right.
$$

\subsection{Evaluation of $s_{1}$ Defined in (19)}

Using the formula

$$
\sum_{n=0}^{N} \epsilon_{n} \cos (n y)=\frac{\sin [(2 N+1) y / 2]}{\sin (y / 2)}
$$

one obtains instead of (19)

$$
\begin{aligned}
& s_{1}=(2 \pi)^{-1} \lim _{N \rightarrow \infty} \int_{0}^{\pi} K_{0}\left[\gamma\left(\rho^{2}+\rho^{\prime 2}-2 \rho \rho^{\prime} \cos x\right)^{1 / 2}\right] \\
& \cdot\left\{\frac{\sin \left[\frac{\pi}{2 \alpha}(2 N+1)(x+\theta)\right.}{\sin \left[\frac{\pi}{2 \alpha}(x+\theta)\right]}+\frac{\sin \left[\frac{\pi}{2 \alpha}(2 N+1)(x-\theta)\right]}{\sin \left[\frac{\pi}{2 \alpha}(x-\theta)\right]}\right\} d x .
\end{aligned}
$$

Substitute in the first integral $\pi(x+\theta)=-2 \alpha t$ and in the second $\pi(x-\theta)=2 \alpha t$. It follows then that

$$
s_{1}=\alpha \pi^{-2} \lim _{N \rightarrow \infty} \int_{t_{1}}^{t_{2}} K_{0}\left\{\gamma\left[\rho^{2}+\rho^{\prime 2}-2 \rho \rho^{\prime} \cos (\theta+2 \alpha t / \pi)\right]\right\}^{1 / 2} \frac{\sin (2 N+1) t}{\sin t} d t,
$$

where $t_{1}=-\frac{\pi}{2 \alpha}(\pi+\theta)$ and $t_{2}=\frac{\pi}{2 \alpha}(\pi-\theta)$. Now $s_{1}$ is a Dirichlet integral and its value follows immediately [2, p. 492] as represented in (22).

Washingtos, D. C., January 14, 1958. 